\title{
Quasi-random sampling for multivariate distributions via generative neural networks
}

\author{
Marius Hofert ${ }^{1}$, Avinash Prasad ${ }^{2}, \mathrm{Mu} \mathrm{Zhu}^{3}$ \\ 2020-04-06
}

\begin{abstract}
Generative moment matching networks (GMMNs) are introduced for generating quasi-random samples from multivariate models with any underlying copula in order to compute estimates under variance reduction. So far, quasi-random sampling for multivariate distributions required a careful design, exploiting specific properties (such as conditional distributions) of the implied parametric copula or the underlying quasi-Monte Carlo (QMC) point set, and was only tractable for a small number of models. Utilizing GMMNs allows one to construct quasi-random samples for a much larger variety of multivariate distributions without such restrictions, including empirical ones from real data with dependence structures not well captured by parametric copulas. Once trained on pseudo-random samples from a parametric model or on real data, these neural networks only require a multivariate standard uniform randomized QMC point set as input and are thus fast in estimating expectations of interest under dependence with variance reduction. Numerical examples are considered to demonstrate the approach, including applications inspired by risk management practice. All results are reproducible with the demos GMMN_QMC_paper, GMMN_QMC_data and GMMN_QMC_timings as part of the $\mathrm{R}$ package gnn.
\end{abstract}

\section{Keywords}

Maximum mean discrepancy, generative moment matching networks, quasi-random numbers, copulas, sums of dependent random variables, expected shortfall.

MSC2010

62H99, 65C60, 60E05, 00A72, 65C10.

\section{Introduction}

Let $\boldsymbol{X}=\left(X_{1}, \ldots, X_{d}\right)$ be a $d$-dimensional random vector with distribution function $F_{\boldsymbol{X}}$ and continuous margins $F_{X_{1}}, \ldots, F_{X_{d}}$. It is not a trivial task in general to generate quasi-random samples $\boldsymbol{X}_{1}, \ldots, \boldsymbol{X}_{n}$ from $F_{\boldsymbol{X}}$, i.e., samples that mimic realizations from $F_{\boldsymbol{X}}$ but preserve low-discrepancy in the sense of being locally more homogeneous with fewer "gaps" or "clusters" (Cambou et al., 2017, Section 4.2).

By Sklar's Theorem, we always have the decomposition

$$
F_{\boldsymbol{X}}(\boldsymbol{x})=C\left(F_{X_{1}}\left(x_{1}\right), \ldots, F_{X_{d}}\left(x_{d}\right)\right), \quad \boldsymbol{x}=\left(x_{1}, \ldots, x_{d}\right) \in \mathbb{R}^{d},
$$

where $C:[0,1]^{d} \rightarrow[0,1]$ is the unique underlying copula (Nelsen, 2006 Joe, 2014). Since, in distribution, $\boldsymbol{X}=F_{\boldsymbol{X}}^{-1}(\boldsymbol{U})$ for $\boldsymbol{U} \sim C$ and $F_{\boldsymbol{X}}^{-1}(\boldsymbol{u})=\left(F_{X_{1}}^{-1}\left(u_{1}\right), \ldots, F_{X_{d}}^{-1}\left(u_{d}\right)\right)$, we shall mostly focus on the problem of generating quasi-random samples $\boldsymbol{U}_{1}, \ldots, \boldsymbol{U}_{n}$ from $C$ rather than $\boldsymbol{X}_{1}, \ldots, \boldsymbol{X}_{n}$ from $F_{\boldsymbol{X}}$, as the latter are easily obtained from the former.

\footnotetext{
${ }^{1}$ Department of Statistics and Actuarial Science, University of Waterloo, 200 University Avenue West, Waterloo, ON, N2L 3G1, marius.hofert@uwaterloo.ca. The author acknowledges support from NSERC (Grant RGPIN-5010-2015).

${ }^{2}$ Department of Statistics and Actuarial Science, University of Waterloo, 200 University Avenue West, Waterloo, ON, N2L 3G1, a2prasad@uwaterloo.ca. The author acknowledges support from NSERC (PGS D Scholarship).

${ }^{3}$ Department of Statistics and Actuarial Science, University of Waterloo, 200 University Avenue West, Waterloo, ON, N2L 3G1, mu.zhu@uwaterloo.ca. The author acknowledges support from NSERC (RGPIN-2016-03876).
} 


\subsection{Existing difficulty}

For the independence copula, $C(\boldsymbol{u})=u_{1} \cdot \ldots \cdot u_{d}$, quasi-random samples can be obtained simply by using randomized quasi-Monte Carlo (RQMC) point sets such as randomized Sobol' or generalized Halton sequences (see, e.g., Lemieux, 2009, Chapter 5).

Recently, Cambou et al. (2017) demonstrated that for a limited number of copulas $C$ (normal, $t$ or Clayton copulas), one can obtain quasi-random samples by transforming RQMC point sets with the inverse Rosenblatt transform of $C$ (Rosenblatt, 1952); this method is known as the conditional distribution method (CDM) - see, e.g., Embrechts et al. (2003) or Hofert (2010, p. 45). Cambou et al. (2017) also showed that transformations to quasi-random copula samples may exist for copulas with a sufficiently simple stochastic representation. For most copulas, the latter is not the case and the CDM is numerically intractable. In other words, there exists no universal and numerically tractable transformation from RQMC point sets to quasi-random samples from copulas. For the majority of copula models, including grouped normal variance mixture copulas, Archimax copulas, nested Archimedean copulas or extreme-value copulas, we simply do not know how to generate quasi-random samples from them.

\subsection{Our contribution}

The main contribution of this paper is to introduce a new approach for quasi-random sampling from $F_{\boldsymbol{X}}$ with any underlying copula $C$, using generative neural networks. Even when we do not know the distribution $F_{\boldsymbol{X}}$, our approach can still provide quasi-random samples from the corresponding empirical distribution $\hat{F}_{\boldsymbol{X}}$ as long as we have a dataset from $\boldsymbol{F}_{\boldsymbol{X}}$. This is especially useful when the dependence structure in the data cannot be adequately captured by a readily available parametric copula; see Section 5 where we present a real-data example to show how useful our approach can be in this case where no adequate copula model is known in the first place.

Specifically, let $f_{\boldsymbol{\theta}}$ denote a neural network $(\mathrm{NN})$ parameterized by $\boldsymbol{\theta}$. We train $f_{\boldsymbol{\theta}}$ so that, given a $p$-dimensional input $\boldsymbol{Z} \sim F_{\boldsymbol{Z}}$ with independent components $Z_{1}, \ldots, Z_{p}$ from known distributions $F_{Z_{1}}, \ldots, F_{Z_{p}}$, the trained $\mathrm{NN}$ can generate $d$-dimensional output from the desired distribution, $f_{\hat{\boldsymbol{\theta}}}(\boldsymbol{Z}) \sim$ $F_{\boldsymbol{X}}$, where $\hat{\boldsymbol{\theta}}$ denotes the parameter vector of the trained NN. We can thus turn a uniform RQMC point set, $\left\{\tilde{\boldsymbol{v}}_{1}, \ldots, \tilde{\boldsymbol{v}}_{n}\right\}$, into a quasi-random sample from $F_{\boldsymbol{X}}$ by letting

$$
\boldsymbol{Y}_{i}=f_{\hat{\boldsymbol{\theta}}} \circ F_{\boldsymbol{Z}}^{-1}\left(\tilde{\boldsymbol{v}}_{i}\right), \quad i=1, \ldots, n,
$$

where $F_{\boldsymbol{Z}}^{-1}(\boldsymbol{u})=\left(F_{Z_{1}}^{-1}\left(u_{1}\right), \ldots, F_{Z_{p}}^{-1}\left(u_{p}\right)\right)$.

\subsection{Assessment}

The theoretical properties of quasi-randomness (or low-discrepancy) under dependence are hard to assess; see Cambou et al. (2017) and Appendix A. In low-dimensional cases (see Section 3.2), we use data visualization tools to assess the quality of the generated quasi-random samples, such as contour plots (or level curves) showing that the empirical copula of our GMMN quasi-random samples is closer to the true target copula than that of GMMN pseudo-random samples. In higher-dimensional cases (see Section 3.3), we use a Cramér-von-Mises goodness-of-fit statistic to make the same point.

Since the main application of quasi-random sampling is to obtain low-variance Monte-Carlo estimates of

$$
\mu=\mathbb{E}(\Psi(\boldsymbol{X}))=\mathbb{E}\left(\Psi\left(F_{\boldsymbol{X}}^{-1}(\boldsymbol{U})\right)\right) \quad \text { for } \quad \boldsymbol{X} \sim F_{\boldsymbol{X}}, \boldsymbol{U} \sim C
$$


for an integrable $\Psi: \mathbb{R}^{d} \rightarrow \mathbb{R}$, we also assess our method in such a specific context. The Monte Carlo estimator approximates this expectation by

$$
\hat{\mu}_{n}^{\mathrm{MC}}=\frac{1}{n} \sum_{i=1}^{n} \Psi\left(F_{\boldsymbol{X}}^{-1}\left(\boldsymbol{U}_{i}\right)\right),
$$

where $\boldsymbol{U}_{1}, \ldots, \boldsymbol{U}_{n} \stackrel{\text { ind. }}{\sim} C$. Using NN-generated quasi-random samples $\boldsymbol{Y}_{1}, \ldots, \boldsymbol{Y}_{n}$ from (2), we can approximate $\mathbb{E}\left(\Psi\left(F_{\boldsymbol{X}}^{-1}(\boldsymbol{U})\right)\right)$ by

$$
\hat{\mu}_{n}^{\mathrm{NN}}=\frac{1}{n} \sum_{i=1}^{n} \Psi\left(\boldsymbol{Y}_{i}\right)=\frac{1}{n} \sum_{i=1}^{n} \Psi\left(f_{\hat{\boldsymbol{\theta}}} \circ F_{\boldsymbol{Z}}^{-1}\left(\tilde{\boldsymbol{v}}_{i}\right)\right) .
$$

Theoretically (Section 2.3 and Appendix A), we establish various guarantees that the estimation error of (3) by (5) will be small as long as both $f_{\hat{\boldsymbol{\theta}}}$ and $\Psi$ are sufficiently smooth; we also establish the corresponding convergence rates. Empirically (Section 4), we verify that (5) indeed has lower variance and converges faster than (4).

Although being the main focus in this paper, let us stress that estimating expectations such as (3) is not the only application of quasi-random sampling. For example, quasi-random sampling can also be used for estimating quantiles for the distribution of a sum of dependent random variables.

All results presented in this paper (and more) are reproducible with the demos GMMN_QMC_paper, GMMN_QMC_data and GMMN_QMC_timings as part of the R package gnn.

\section{Quasi-random GMMN samples}

\subsection{Generative moment matching networks}

In this paper, we work with the multi-layer perceptron $(M L P)$, which is regarded as the quintessential neural network $(N N)$. Let $L$ be the number of (hidden) layers in the $\mathrm{NN}$ and, for each $l=0, \ldots, L+1$, let $d_{l}$ be the dimension of layer $l$, that is, the number of neurons in layer $l$. In this notation, layer $l=0$ refers to the input layer which consists of the input $\boldsymbol{z} \in \mathbb{R}^{p}$ for $d_{0}=p$, and layer $l=L+1$ refers to the output layer which consists of the output $\boldsymbol{y} \in \mathbb{R}^{d}$ for $d_{L+1}=d$. Layers $l=1, \ldots, L+1$ can be described in terms of the output $\boldsymbol{a}_{l-1} \in \mathbb{R}^{d_{l-1}}$ of layer $l-1$ via

$$
\begin{aligned}
\boldsymbol{a}_{0} & =\boldsymbol{z} \in \mathbb{R}^{d_{0}}, \\
\boldsymbol{a}_{l} & =f_{l}\left(\boldsymbol{a}_{l-1}\right)=\phi_{l}\left(W_{l} \boldsymbol{a}_{l-1}+\boldsymbol{b}_{l}\right) \in \mathbb{R}^{d_{l}}, \quad l=1, \ldots, L+1, \\
\boldsymbol{y} & =\boldsymbol{a}_{L+1} \in \mathbb{R}^{d_{L+1}},
\end{aligned}
$$

with weight matrices $W_{l} \in \mathbb{R}^{d_{l} \times d_{l-1}}$, bias vectors $\boldsymbol{b}_{l} \in \mathbb{R}^{d_{l}}$ and activation functions $\phi_{l}$; note that for vector inputs the activation function $\phi_{l}$ is understood to be applied componentwise.

The NN $f_{\boldsymbol{\theta}}: \mathbb{R}^{p} \rightarrow \mathbb{R}^{d}$ can then be written as the composition

$$
f_{\boldsymbol{\theta}}=f_{L+1} \circ f_{L} \circ \cdots \circ f_{2} \circ f_{1},
$$

with its (flattened) parameter vector given by $\boldsymbol{\theta}=\left(W_{1}, \ldots, W_{L+1}, \boldsymbol{b}_{1}, \ldots, \boldsymbol{b}_{L+1}\right)$. To fit $\boldsymbol{\theta}$, we use the backpropagation algorithm (a stochastic gradient descent) based on a cost function E. Conceptually, $E$ computes a distance between the target output $\boldsymbol{x} \in \mathbb{R}^{d}$ and the actual output $\boldsymbol{y}=\boldsymbol{y}(\boldsymbol{z}) \in \mathbb{R}^{d}$ predicted by the NN; what is actually computed is a sample version of $E$ based on a subsample (the so-called mini-batch), see Section 2.2 . 
The expressive power of NNs is primarily characterized by the universal approximation theorem; see Goodfellow et al. (2016, Chapter 6). In particular, given suitable activation functions, a single hidden layer NN with a finite number of neurons can approximate any continuous function on a compact subset of the multidimensional Euclidean space; see Nielsen (2015, Chapter 4) for a visual account of the validity of the universal approximation theorem. Cybenko (1989) first proposed such universal approximation results for the sigmoid activation function $\phi_{l}(x)=1 /\left(1+\mathrm{e}^{-x}\right)$ and Hornik $(1991$, Theorem 1) then generalized the results to include arbitrary bounded and non-constant activation functions. In recent years, the rectified linear unit (ReLU) $\phi_{l}(x)=\max \{0, x\}$ has become the most popular activation function for efficiently training NNs. This unbounded activation function does not satisfy the assumptions of the universal approximation theorem in Hornik (1991). However, there have since been numerous theoretical investigations into the expressive power of NNs with ReLU activation functions; see, for example, Pascanu et al. (2013), Montufar et al. (2014) or R. Arora et al. (2016). In particular, for certain conditions on the number of layers and neurons in the NN, R. Arora et al. (2016) provide a similar universal approximation theorem for NNs with ReLU activation functions.

Li et al. (2015) and Dziugaite et al. (2015) simultaneously introduced a type of generative neural network known as the generative moment matching network (GMMN) or the Maximum Mean Discrepancy (MMD) net. GMMNs are NNs $f_{\boldsymbol{\theta}}$ of the above form which utilize a (kernel) maximum mean discrepancy statistic as the cost function (see later). Conceptually, they can be thought of as parametric maps of a given sample $\boldsymbol{Z}=\left(Z_{1}, \ldots, Z_{p}\right)$ from an input distribution $F_{\boldsymbol{Z}}$ to a sample $\boldsymbol{X}=\left(X_{1}, \ldots, X_{d}\right)$ from the target distribution $F_{\boldsymbol{X}}$. As is standard in the literature, we assume independence among the components of $\boldsymbol{Z}=\left(Z_{1}, \ldots, Z_{p}\right)$. Typical choices for the distribution of the $Z_{j}$ 's are $\mathrm{U}(0,1)$ or $\mathrm{N}(0,1)$. The objective is then to generate samples from the target distribution via the trained GMMN $f_{\hat{\boldsymbol{\theta}}}$. The MMD nets introduced in Dziugaite et al. (2015) are almost identical to GMMNs but with a slight difference in the training procedure; additionally, Dziugaite et al. (2015) provided a theoretical framework for analyzing optimization algorithms with (kernel) MMD cost functions.

\subsection{Cost function and training of GMMNs}

To learn $f_{\boldsymbol{\theta}}$ (or, statistically speaking, to estimate the parameter vector $\boldsymbol{\theta}$ ) we assume that we have $n_{\text {trn }}$ training data points $\boldsymbol{X}_{1}, \ldots, \boldsymbol{X}_{n_{\text {trn }}}$ from $\boldsymbol{X}$, either in the form of a pseudo-random sample from $F_{\boldsymbol{X}}$ or as real data. Based on a sample $\boldsymbol{Z}_{1}, \ldots, \boldsymbol{Z}_{n_{\text {trn }}}$ from the input distribution, the GMMN generates the output sample $\boldsymbol{Y}_{1}, \ldots, \boldsymbol{Y}_{n_{\text {trn }}}$, where $\boldsymbol{Y}_{i}=f_{\boldsymbol{\theta}}\left(\boldsymbol{Z}_{i}\right), i=1, \ldots, n_{\operatorname{trn}}$. Stacking $\boldsymbol{X}_{1}, \ldots, \boldsymbol{X}_{n_{\text {trn }}}$ into an $n_{\text {trn }} \times d$ matrix $X$ and likewise $\boldsymbol{Y}_{1}, \ldots, \boldsymbol{Y}_{n_{\text {trn }}}$ into $Y$, we are thus interested in whether the two samples $X$ and $Y$ come from the same distribution.

To this end, GMMNs utilize as cost function $E$ the maximum mean discrepancy (MMD) statistic from the kernel two-sample test introduced by Gretton et al. (2007), whose sample version is given by

$$
\operatorname{MMD}(X, Y)=\sqrt{\frac{1}{n_{\text {trn }}^{2}} \sum_{i_{1}=1}^{n_{\text {trn }}} \sum_{i_{2}=1}^{n_{\text {trn }}}\left(K\left(\boldsymbol{X}_{i_{1}}, \boldsymbol{X}_{i_{2}}\right)-2 K\left(\boldsymbol{X}_{i_{1}}, \boldsymbol{Y}_{i_{2}}\right)+K\left(\boldsymbol{Y}_{i_{1}}, \boldsymbol{Y}_{i_{2}}\right)\right)}
$$

where $K(\cdot, \cdot): \mathbb{R}^{d} \times \mathbb{R}^{d} \rightarrow \mathbb{R}$ denotes a kernel (similarity) function. If $K(\cdot, \cdot)$ is a so-called universal kernel function (e.g., Gaussian or Laplace), then it can be shown (Gretton et al., 2007; Gretton et al., 2012 that the MMD converges in probability to 0 for $n_{\operatorname{trn}} \rightarrow \infty$ if and only if $\boldsymbol{Y}=\boldsymbol{X}$ in distribution. This makes the MMD with a universal kernel function an intuitive choice as cost function for training $f_{\boldsymbol{\theta}}$ to learn a random number generator from a multivariate distribution $F_{\boldsymbol{X}}$; our choice of kernel $K$ is addressed in Section 3.1. For the Gaussian kernel in particular, expanding the exponential, one can see that minimizing the MMD can be interpreted as matching all the moments of the distributions of $\boldsymbol{X}$ 
and $\boldsymbol{Y}$ which gives an interpretation of the MMD in this case. Note that, in comparison to a quadratic cost function, all pairs of observations enter the MMD, which turns out to be a crucial property for learning a random number generator of $F_{\boldsymbol{X}}$.

Computing $\operatorname{MMD}(X, Y)$ in (6) requires one to evaluate the kernel for all $\left(\begin{array}{c}n_{\operatorname{trn}} \\ 2\end{array}\right)$ pairs of observations, which is memory-prohibitive for even moderately large $n_{\operatorname{trn}}$. As suggested by Li et al. (2015), we thus adopt a mini-batch optimization procedure. Instead of directly optimizing the MMD for the entire training dataset, we partition the data into batches of size $n_{\text {bat }}$ and use the batches sequentially to update the parameters $\boldsymbol{\theta}$ of the GMMN with the Adam optimizer of Kingma and Ba (2014). Rather than following the gradient at each iterative step, the Adam optimizer essentially uses a "memorysticking gradient" - a weighted combination of the current gradient and past gradients from earlier iterations. After all the training data are exhausted, i.e., roughly after $\left(n_{\text {trn }} / n_{\text {bat }}\right)$-many batches or gradient steps, one epoch of the training of the GMMN is completed. The overall training procedure is considered completed after $n_{\text {epo }}$ epochs. The training of the GMMN can thus be summarized as follows:

\section{Algorithm 2.1 (Training GMMNs)}

1) Fix the number $n_{\text {epo }}$ of epochs and the batch size $1 \leq n_{\text {bat }} \leq n_{\text {trn }}$ per epoch, where $n_{\text {bat }}$ is assumed to divide $n_{\text {trn }}$. Initialize the epoch counter $k=0$ and the GMMN's parameter vector $\boldsymbol{\theta}$; we follow Glorot and Bengio (2010) and initialize the components of $\boldsymbol{\theta}$ as $W_{l} \sim$ $\mathrm{U}\left(-\sqrt{6 /\left(d_{l}+d_{l-1}\right)}, \sqrt{6 /\left(d_{l}+d_{l-1}\right)}\right)^{d_{l}} \times d_{l-1}$ and $\boldsymbol{b}_{l}=\mathbf{0}$ for $l=1, \ldots, L+1$.

2) For epoch $k=1, \ldots, n_{\text {epo }}$, do:

2.1) Randomly partition the input distribution sample $\boldsymbol{Z}_{1}, \ldots, \boldsymbol{Z}_{n_{\operatorname{trn}}}$ and training sample $\boldsymbol{X}_{1}, \ldots, \boldsymbol{X}_{n_{\operatorname{trn}}}$ into corresponding $n_{\text {trn }} / n_{\text {bat }}$ non-overlapping batches $\boldsymbol{Z}_{1}^{(b)}, \ldots, \boldsymbol{Z}_{n_{\text {bat }}}^{(b)}$ and $\boldsymbol{X}_{1}^{(b)}, \ldots, \boldsymbol{X}_{n_{\text {bat }}}^{(b)}$, $b=1, \ldots, n_{\text {trn }} / n_{\text {bat }}$, of size $n_{\text {bat }}$ each.

2.2) For batch $b=1, \ldots, n_{\text {trn }} / n_{\text {bat }}$, do:

2.2.1) Compute the GMMN output $\boldsymbol{Y}_{i}^{(b)}=f_{\boldsymbol{\theta}}\left(\boldsymbol{Z}_{i}^{(b)}\right), i=1, \ldots, n_{\text {bat. }}$.

2.2.2) Compute the gradient $\frac{\partial}{\partial \boldsymbol{\theta}} \operatorname{MMD}\left(X^{(b)}, Y^{(b)}\right)$ from the samples $X^{(b)}\left(\operatorname{stacking} \boldsymbol{X}_{1}^{(b)}, \ldots, \boldsymbol{X}_{n_{\text {bat }}}^{(b)}\right)$ and $Y^{(b)}$ (stacking $\boldsymbol{Y}_{1}^{(b)}, \ldots, \boldsymbol{Y}_{n_{\text {bat }}^{(b)}}$ ) via automatic differentiation.

2.2.3) Take a gradient step to update $\boldsymbol{\theta}$ with the Adam optimizer popularized by Kingma and Ba (2014, Algorithm 1).

3) Return $\hat{\boldsymbol{\theta}}=\boldsymbol{\theta}$; the fitted GMMN is then $f_{\hat{\boldsymbol{\theta}}}$.

\subsection{Pseudo- and quasi-random sampling by GMMN}

The following algorithm describes how to obtain a pseudo-random sample of $\boldsymbol{Y}$ via the trained GMMN $f_{\hat{\boldsymbol{\theta}}}$ from a pseudo-random sample $\boldsymbol{Z} \sim F_{\boldsymbol{Z}}$.

\section{Algorithm 2.2 (Pseudo-random sampling by GMMN)}

1) Fix the number $n_{\text {gen }}$ of samples to generate from $\boldsymbol{Y}$.

2) Draw $\boldsymbol{Z}_{i} \stackrel{\text { ind. }}{\sim} F_{\boldsymbol{Z}}, i=1, \ldots, n_{\text {gen }}$, for example, via $\boldsymbol{Z}_{i}=F_{\boldsymbol{Z}}^{-1}\left(\boldsymbol{U}_{i}^{\prime}\right), i=1, \ldots, n_{\text {gen }}$, where $\boldsymbol{U}_{1}^{\prime}, \ldots, \boldsymbol{U}_{n_{\text {gen }}}^{\prime} \stackrel{\text { ind. }}{\sim} \mathrm{U}(0,1)^{p}$

3) Return $\boldsymbol{Y}_{i}=f_{\hat{\boldsymbol{\theta}}}\left(\boldsymbol{Z}_{i}\right), i=1, \ldots, n_{\text {gen }}$; to obtain a sample from $C$, return the pseudo-observations of $\boldsymbol{Y}_{1}, \ldots, \boldsymbol{Y}_{n_{\text {gen }}}$ (Genest, Ghoudi, et al., 1995). 


\section{Quasi-random GMMN samples}

To obtain quasi-random samples from $F_{\boldsymbol{X}}$ with underlying copula $C$, we replace $\boldsymbol{U}_{1}^{\prime}, \ldots, \boldsymbol{U}_{n_{\text {gen }}}^{\prime} \stackrel{\text { ind. }}{\sim}$ $\mathrm{U}(0,1)^{p}$ in Algorithm 2.2 by an RQMC point set to obtain the following algorithm; the randomization is done to obtain unbiased QMC estimators and estimates of their variances.

\section{Algorithm 2.3 (Quasi-random sampling by GMMN)}

1) Fix the number $n_{\text {gen }}$ of samples to generate from $\boldsymbol{Y}$.

2) Compute an RQMC point set $\tilde{P}_{n_{\text {gen }}}=\left\{\tilde{\boldsymbol{v}}_{1}, \ldots, \tilde{\boldsymbol{v}}_{n_{\text {gen }}}\right\}$ (for example, a randomized Sobol' or a generalized Halton sequence) and $\boldsymbol{Z}_{i}=F_{\boldsymbol{Z}}^{-1}\left(\tilde{\boldsymbol{v}}_{i}\right), i=1, \ldots, n_{\text {gen }}$.

3) Return $\boldsymbol{Y}_{i}=f_{\hat{\boldsymbol{\theta}}}\left(\boldsymbol{Z}_{i}\right), i=1, \ldots, n_{\text {gen }}$; to obtain a sample from $C$, return the pseudo-observations of $\boldsymbol{Y}_{1}, \ldots, \boldsymbol{Y}_{n_{\text {gen }}}$.

Note that $\tilde{P}_{n_{\text {gen }}}$ mimics $\mathrm{U}(0,1)^{p}$, and not $C$. As mentioned in the introduction, Cambou et al. (2017) presented transformations to convert $\tilde{P}_{n_{\text {gen }}}$ to samples which mimic samples from $C$ but locally provide a more homogeneous coverage. Unfortunately, these transformations are only available for a few specific cases of $C$ and their numerical evaluation in a fast and robust way is even more challenging.

To avoid these problems, we suggest to utilize the GMMN $f_{\hat{\boldsymbol{\theta}}}$ trained as in Algorithm 2.1. Besides a straightforward evaluation, this allows us to generate quasi-random samples from $F_{\boldsymbol{X}}$ with any underlying copula $C$, by training a GMMN on pseudo-random samples generated from $F_{\boldsymbol{X}}$. Alternatively, quasi-random samples which follow the same empirical distribution as any given dataset can be obtained by training a GMMN on the given dataset itself. An additional advantage is that GMMNs provide a sufficiently smooth map from the RQMC point set to the target distribution which helps preserve the low-discrepancy of the point set upon transformation and hence guarantees the improved performance of RQMC estimators compared to the MC estimator (see Section 4 and Appendix A).

With the mapping $F_{Z}^{-1}(\boldsymbol{u})=\left(F_{Z_{1}}^{-1}\left(u_{1}\right), \ldots, F_{Z_{p}}^{-1}\left(u_{p}\right)\right)$ to the input distribution and the trained GMMN $f_{\hat{\boldsymbol{\theta}}}$ at hand, define a transform

$$
q(\boldsymbol{u})=f_{\hat{\boldsymbol{\theta}}} \circ F_{\boldsymbol{Z}}^{-1}(\boldsymbol{u}), \quad \boldsymbol{u} \in(0,1)^{p} .
$$

Based on the RQMC point set $\tilde{P}_{n_{\text {gen }}}=\left\{\tilde{\boldsymbol{v}}_{1}, \ldots, \tilde{\boldsymbol{v}}_{n_{\text {gen }}}\right\}$ of size $n_{\text {gen }}$, we can then obtain quasi-random samples by

$$
\boldsymbol{Y}_{i}=q\left(\tilde{\boldsymbol{v}}_{i}\right), \quad i=1, \ldots, n_{\mathrm{gen}},
$$

(compare with (2) ) and define a GMMN RQMC estimator of (3) by

$$
\hat{\mu}_{n_{\text {gen }}}^{\mathrm{NN}}=\frac{1}{n_{\text {gen }}} \sum_{i=1}^{n_{\text {gen }}} \Psi\left(\boldsymbol{Y}_{i}\right)=\frac{1}{n_{\text {gen }}} \sum_{i=1}^{n_{\text {gen }}} \Psi\left(q\left(\tilde{\boldsymbol{v}}_{i}\right)\right)=\frac{1}{n_{\text {gen }}} \sum_{i=1}^{n_{\text {gen }}} \Psi\left(f_{\hat{\boldsymbol{\theta}}}\left(F_{\boldsymbol{Z}}^{-1}\left(\tilde{\boldsymbol{v}}_{i}\right)\right)\right) .
$$

We thus have the approximations

$$
\mathbb{E}(\Psi(\boldsymbol{X})) \approx \mathbb{E}(\Psi(\boldsymbol{Y})) \approx \hat{\mu}_{n_{\mathrm{gen}}}^{\mathrm{NN}}
$$

The error in the first approximation is small if the GMMN is trained well and the error in the second approximation is small if the unbiased estimator $\hat{\mu}_{n_{\mathrm{gen}}}^{\mathrm{NN}}$ has a small variance. The primary bottleneck in this setup is the error in the first approximation which is determined by the size $n_{\text {trn }}$ of the training dataset and, in particular, by the batch size $n_{\text {bat }}$ which is the major factor determining training efficiency of the GMMN we found in all our numerical studies. Given a sufficiently large $n_{\text {bat }}$ and, by extension, $n_{\text {trn }}$, the GMMN is trained well, which renders the first approximation error in (8) 
negligible. However, in practice the batch size $n_{\text {bat }}$ is constrained by the quadratically increasing memory demands to compute the MMD cost function of the GMMN. For a theoretical result regarding this approximation error, see Dziugaite et al. (2015) where a bound on the error between optimizing a sample version and a population version of $\operatorname{MMD}(X, Y)$ was investigated. Finally, let us note that the task of GMMN training and generation are separate steps which ensures that, once trained, generating quasi-random GMMN samples is comparably fast; see Appendix B.

The error in the second approximation in (8) is small if the composite function $\Psi \circ q$ is sufficiently smooth. The transform $q$ is sufficiently smooth for GMMNs $f_{\hat{\boldsymbol{\theta}}}$ constructed using standard activation functions and commonly used input distributions; see the discussion following Corollary A.4. Given a sufficiently smooth $\Psi$, we can establish a rate of convergence $O\left(n_{\text {gen }}^{-3}\left(\log n_{\text {gen }}\right)^{p-1}\right)$ for the variance (and $O\left(n_{\text {gen }}^{-3 / 2}\left(\log n_{\text {gen }}\right)^{(p-1) / 2}\right)$ for the approximation error) of the GMMN RQMC estimator $\hat{\mu}_{n_{\text {gen }}}^{\mathrm{NN}}$ constructed using scrambling as randomization; see Appendix A.4.1. With a stronger assumption on the behavior of the composite function $\Psi \circ q$, we can show that the Koksma-Hlawka bound on the error between the (non-randomized) GMMN QMC estimator $\frac{1}{n_{\text {gen }}} \sum_{i=1}^{n_{\text {gen }}} \Psi\left(q\left(\boldsymbol{v}_{i}\right)\right)$ and $\mathbb{E}(\Psi(\boldsymbol{Y}))$ is satisfied which in turn implies a rate of convergence $O\left(n_{\text {gen }}^{-1}\left(\log n_{\text {gen }}\right)^{p}\right)$ for the (non-randomized) GMMN QMC estimator; see Appendix A.2. If the Koksma-Hlawka bound holds, we can also establish a rate of convergence $O\left(n_{\text {gen }}^{-2}\left(\log n_{\text {gen }}\right)^{2 p}\right)$ for the variance of GMMN RQMC estimators constructed using the digital shift method as randomization technique; see Appendix A.4.2

\section{GMMN pseudo- and quasi-random samples for copula models}

In this section we assess the quality of pseudo-random samples and quasi-random samples generated from GMMNs. In both cases we train GMMNs on pseudo-random samples $\boldsymbol{U}_{1}, \ldots, \boldsymbol{U}_{n_{\operatorname{trn}}} \sim C$ from the respective copula $C$. We start by addressing key implementation details and hyperparameters of Algorithm 2.1 that we used in all examples thereafter. By utilizing this algorithm to train $f_{\boldsymbol{\theta}}$ for a wide variety of copula families, we then investigate the quality of the samples $\boldsymbol{Y}_{1}, \ldots, \boldsymbol{Y}_{n_{\text {gen }}}$, once generated by Algorithm 2.2 and once by Algorithm 2.3.

\subsection{GMMN architecture, choice of kernel and training setup}

We find a single hidden layer architecture $(L=1)$ to be sufficient for all the examples we considered. This is because, in this paper, we largely consider the cases of $d \in\{2, \ldots, 10\}$. Learning an entire distribution nonparametrically for $d>10$ would most likely require $L>1$, but it would also require a much larger sample size $n_{\text {trn }}$ and become much more challenging computationally for GMMNs recall from Section 2.2 that the cost function requires $\left(\begin{array}{c}n_{\text {trn }} \\ 2\end{array}\right)$ evaluations. After experimentation, we fix $d_{1}=300, \phi_{1}$ to be ReLU (it offers computational efficiency via non-expensive and non-vanishing gradients) and $\phi_{2}$ to be sigmoid (to obtain outputs in $[0,1]^{d}$ ).

To avoid the need of fine-tuning the bandwidth parameter, we follow Li et al. (2015) and use a mixture of Gaussian kernels with different bandwidth parameters as our kernel function for the MMD statistic in (6); specifically,

$$
K(\boldsymbol{x}, \boldsymbol{y})=\sum_{i=1}^{n_{\mathrm{krn}}} K\left(\boldsymbol{x}, \boldsymbol{y} ; \sigma_{i}\right),
$$

where $n_{\text {krn }}$ denotes the number of mixture components and $K(\boldsymbol{x}, \boldsymbol{y} ; \sigma)=\exp \left(-\|\boldsymbol{x}-\boldsymbol{y}\|_{2}^{2} /\left(2 \sigma^{2}\right)\right)$ is the Gaussian kernel with bandwidth parameter $\sigma>0$. After experimentation, we fix $n_{\mathrm{krn}}=6$ and choose $\left(\sigma_{1}, \ldots, \sigma_{6}\right)=(0.001,0.01,0.15,0.25,0.50,0.75)$; note that copula samples are in $[0,1]^{d}$. 
Unless otherwise specified, we use the following setup across all examples. We use $n_{\text {trn }}=60000$ training data points and find this to be sufficiently large to obtain reliable $f_{\hat{\boldsymbol{\theta}}}$. As dimension of the input distribution $F_{\boldsymbol{Z}}$, we choose $p=d$, that is, the GMMN $f_{\boldsymbol{\theta}}$ is set to be a $d$-to- $d$ transformation. For $F_{\boldsymbol{Z}}$, we choose $\boldsymbol{Z} \sim \mathrm{N}\left(\mathbf{0}, I_{d}\right)$, where $I_{d}$ denotes the identity matrix in $\mathbb{R}^{d \times d}$, so $\boldsymbol{Z}$ consists of independent standard normal random variables; this choice worked better than $\mathrm{U}(0,1)^{d}$ in practice despite the fact that $\mathrm{N}\left(\mathbf{0}, I_{d}\right)$ does not satisfy the assumptions of Proposition A.1. We choose a batch size of $n_{\text {bat }}=5000$ in Algorithm 2.1 this decision is motivated from a practical trade-off that a small $n_{\text {bat }}$ will lead to poor estimates of the population MMD cost function but a large $n_{\text {bat }}$ will incur quadratically growing memory requirements due to (6). As the number of epochs we choose $n_{\text {epo }}=300$ which is generally sufficient in our experiments to obtain accurate results. The tuning parameters of the Adam optimizer is set to the default values reported in Kingma and Ba (2014).

All results in this section, Section 4 and Appendix A.4.2 are reproducible with the demo GMMN_QMC_paper of the R package gnn. Our implementation utilizes the $R$ packages keras and tensorflow which serve as $\mathrm{R}$ interfaces to the corresponding namesake Python libraries. Furthermore, all GMMN training is carried out on one NVIDIA Tesla P100 GPU. To generate the RQMC point set in Algorithm 2.3. we use scrambled nets (Owen, 1995); see also Appendix A.3. Specifically, we use the implementation sobol (, randomize $=$ "Owen") from the R package qrng. Finally, our choice of $\mathrm{R}$ as programming language for this work was motivated by the fact that contributed packages providing functionality for copula modeling and quasi-random number generation — two of the three major fields of research (besides machine learning) this work touches upon - exist in R.

\subsection{Visual assessments of GMMN samples}

In this section we primarily focus on the bivariate case but include an example involving a trivariate copula; for higher-dimensional copulas, see Sections 3.3 and 4. For all one-parameter copulas considered, the single parameter will be chosen such that Kendall's tau, denoted by $\tau$, is equal to 0.25 (weak dependence), 0.50 (moderate dependence) or 0.75 (strong dependence); clearly, this only applies to copula families where there is a one-to-one mapping between the copula parameter and $\tau$.

\subsection{1 $t$, Archimedean copulas and their associated mixtures}

First, we consider Student $t$ copulas, Archimedean copulas, and their mixtures.

Student $t$ copulas are prominent members of the elliptical class of copulas and are given by $C(\boldsymbol{u})=t_{\nu, P}\left(t_{\nu}^{-1}\left(u_{1}\right), \ldots, t_{\nu}^{-1}\left(u_{d}\right)\right), \boldsymbol{u} \in[0,1]^{d}$, where $t_{\nu, P}$ denotes the distribution function of the $d$-dimensional $t$ distribution with $\nu$ degrees of freedom, location vector $\mathbf{0}$ and correlation matrix $P$, and $t_{\nu}^{-1}$ denotes the quantile function of the univariate $t$ distribution with $\nu$ degrees of freedom. For all $t$ copulas considered in this work, we fix $\nu=4$. Student $t$ copulas have explicit inverse Rosenblatt transforms, so one can utilize the CDM for generating quasi-random samples from them (Cambou et al., 2017).

Archimedean copulas are copulas of the form

$$
C(\boldsymbol{u})=\psi\left(\psi^{-1}\left(u_{1}\right), \ldots, \psi^{-1}\left(u_{d}\right)\right), \quad \boldsymbol{u} \in[0,1]^{d},
$$

for an Archimedean generator $\psi$ which is a continuous, decreasing function $\psi:[0, \infty] \rightarrow[0,1]$ that satisfies $\psi(0)=1, \psi(\infty)=\lim _{t \rightarrow \infty} \psi(t)=0$ and that is strictly decreasing on $[0, \inf t: \psi(t)=0]$. Examples of Archimedean generators include $\psi_{C}(t)=(1+t)^{-1 / \theta}($ for $\theta>0)$ and $\psi_{G}(t)=\exp \left(-t^{1 / \theta}\right)$ (for $\theta \geq 1$ ), generating Clayton and Gumbel copulas, respectively. While the inverse Rosenblatt transform and thus the CDM is available analytically for Clayton copulas, this is not the case for 
Gumbel; in Appendix B we used numerical root finding to include the latter case for the purpose of timings only.

We additionally consider equally-weighted two-component mixture copulas in which one component is a 90-degree-rotated $t_{4}$ copula with $\tau=0.50$ and the other component is either a Clayton copula $(\tau=0.50)$ or a Gumbel copula $(\tau=0.50)$. The two mixture copula models are referred to as Clayton- $t(90)$ and Gumbel- $t(90)$ copulas, respectively.

The top rows of Figures 13 display contour plots of true $t$, Clayton and Gumbel copulas respectively, with $\tau=0.25$ (left), 0.50 (middle) and 0.75 (right) along with contours of empirical copulas based on GMMN pseudo-random and GMMN quasi-random samples corresponding to each true copula $C$. The top row of Figure 4 displays similar plots for Clayton- $t(90)$ (left) and Gumbel- $t$ (90) (right) copulas. In each plot, across all figures described above, we observe that the contour of the empirical copula based on GMMN pseudo-random samples is visually fairly similar to the contour of $C$, thus indicating that the 11 GMMNs have been trained sufficiently well. We also see that the contours of the empirical copulas based on GMMN quasi-random samples better approximate the contours of $C$ than the contours of the empirical copulas based on the corresponding pseudo-random samples. This observation indicates that, at least visually, the 11 GMMN transforms (corresponding to each $C$ ) have preserved the low-discrepancy of the input RQMC point sets.

The bottom rows of Figures 14 display Rosenblatt transformed GMMN quasi-random samples, corresponding to each of the 11 true copulas $C$ under consideration. The Rosenblatt transform for a bivariate copula $C$ maps $\left(U_{1}, U_{2}\right) \sim C$ to $\left(R_{1}, R_{2}\right)=\left(U_{1}, C_{2 \mid 1}\left(U_{2} \mid U_{1}\right)\right)$, where $C_{2 \mid 1}\left(u_{2} \mid u_{1}\right)$ denotes the conditional distribution function of $U_{2}$ given $U_{1}=u_{1}$ under $C$. We exploit the fact that $\left(R_{1}, R_{2}\right) \sim \mathrm{U}(0,1)^{2}$ if and only if $\left(U_{1}, U_{2}\right) \sim C$. Moreover, Rosenblatt transforming the GMMN quasi-random samples should yield a more homogeneous coverage of $[0,1]^{2}$. From each of the scatter plots in Figures 14 , we observe no significant departure from $\mathrm{U}(0,1)^{2}$, thus indicating that the GMMNs have learned sufficient approximations to the corresponding true copulas $C$. Furthermore, the lack of gaps or clusters in the scatter plots provides some visual confirmation of the low-discrepancy of the Rosenblatt-transformed GMMN quasi-random samples.

\subsubsection{Nested Archimedean, Marshall-Olkin and mixture copulas}

Next, we consider more complex copulas such as nested Archimedean copulas and Marshall-Olkin copulas. We also re-consider the two mixture copulas introduced in the previous section along with an additional mixture copula. To better showcase the complexity of these dependence structures, we use scatter plots instead of contour plots to display copula and GMMN-generated samples. We omit the plots containing the Rosenblatt transformed samples since they are harder to obtain for the copulas we investigate in this section.

Nested Archimedean copulas (NACs) are Archimedean copulas with arguments possibly replaced by other NACs; see McNeil (2008) or Hofert (2012). In particular, this class of copulas allows us to construct asymmetric extensions of Archimedean copulas. Important to note here is that NACs are copulas for which there is no known (tractable) CDM. To demonstrate the ability of GMMNs to capture such dependence structures, we consider the simplest three-dimensional copula for visualization and investigate higher-dimensional NACs in Sections 3.3 and 4 . The three-dimensional NAC we consider here is

$$
C(\boldsymbol{u})=C_{0}\left(C_{1}\left(u_{1}, u_{2}\right), u_{3}\right), \quad \boldsymbol{u} \in[0,1]^{3},
$$

where $C_{0}$ is a Clayton copula with Kendall's tau $\tau_{0}=0.25$ and $C_{1}$ is a Clayton copula with Kendall's tau $\tau_{1}=0.50$. In Sections 3.3 and 4 , we will present examples of five- and ten-dimensional NACs. 
3 GMMN pseudo- and quasi-random samples for copula models
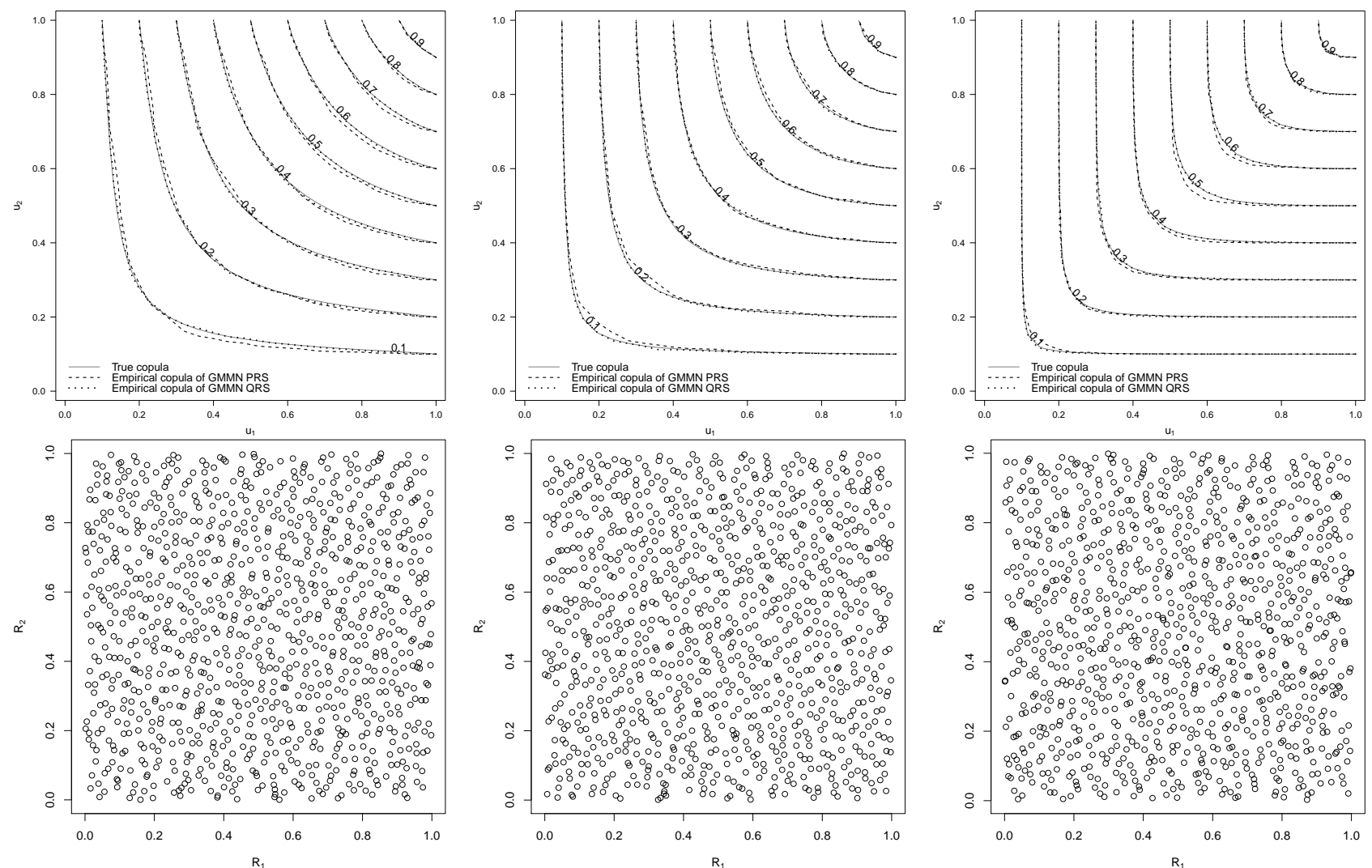

Figure 1 Top row contains contour plots of true $t_{4}$ copulas with $\tau=0.25$ (left), 0.50 (middle) and 0.75 (right) along with the corresponding contour plots of empirical copulas based on GMMN pseudo-random and GMMN quasi-random samples (respectively, GMMN PRS and GMMN QRS), both of size $n_{\text {gen }}=1000$. Bottom row contains Rosenblatt-transformed GMMN QRS corresponding to the same three $t_{4}$ copulas. 
3 GMMN pseudo- and quasi-random samples for copula models
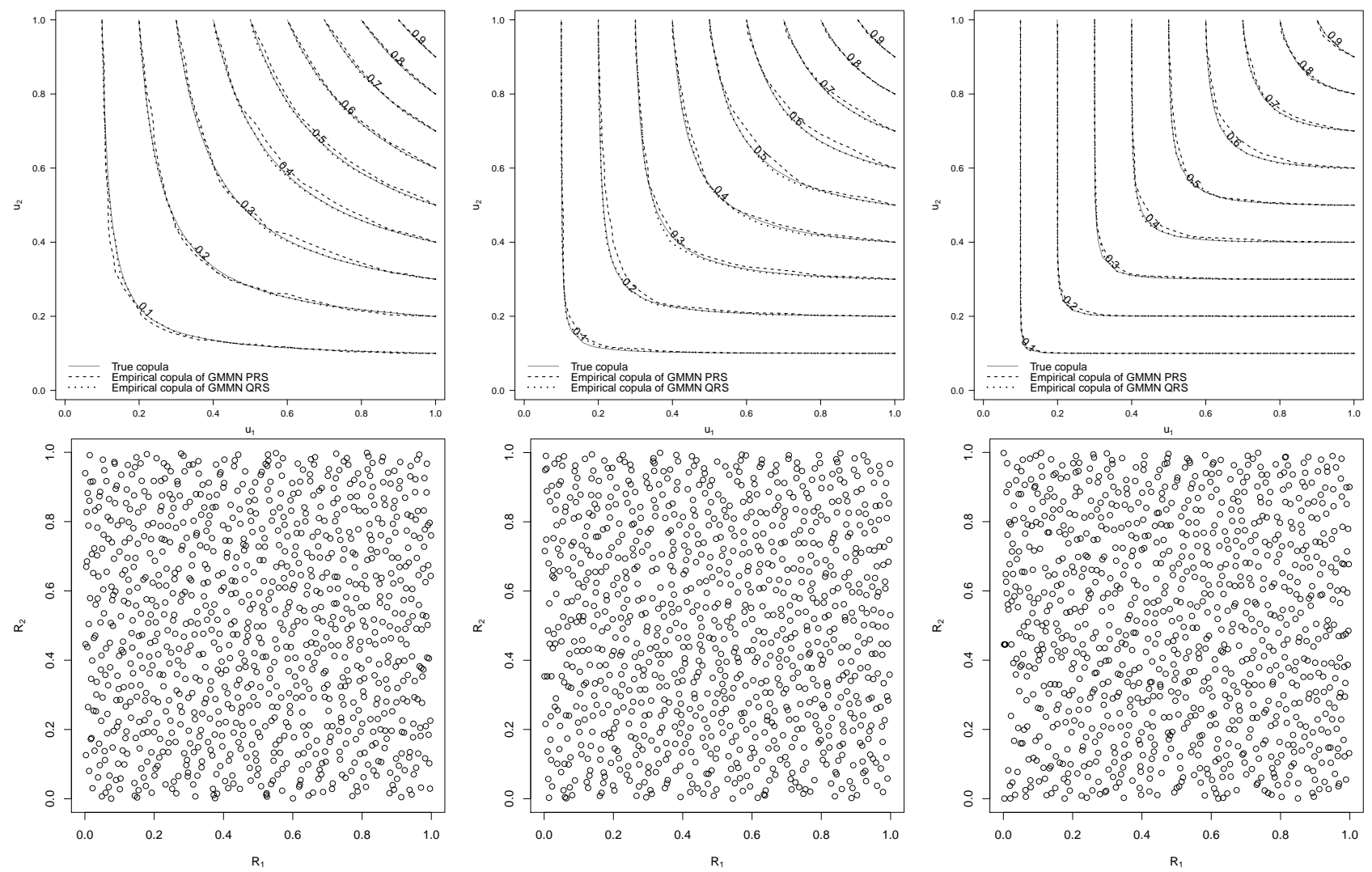

Figure 2 Top row contains contour plots of true Clayton copulas with $\tau=0.25$ (left), 0.50 (middle) and 0.75 (right) along with the corresponding contour plots of empirical copulas based on GMMN PRS and GMMN QRS, both of size $n_{\text {gen }}=1000$. Bottom row contains Rosenblatttransformed GMMN QRS corresponding to the same three Clayton copulas. 
3 GMMN pseudo- and quasi-random samples for copula models
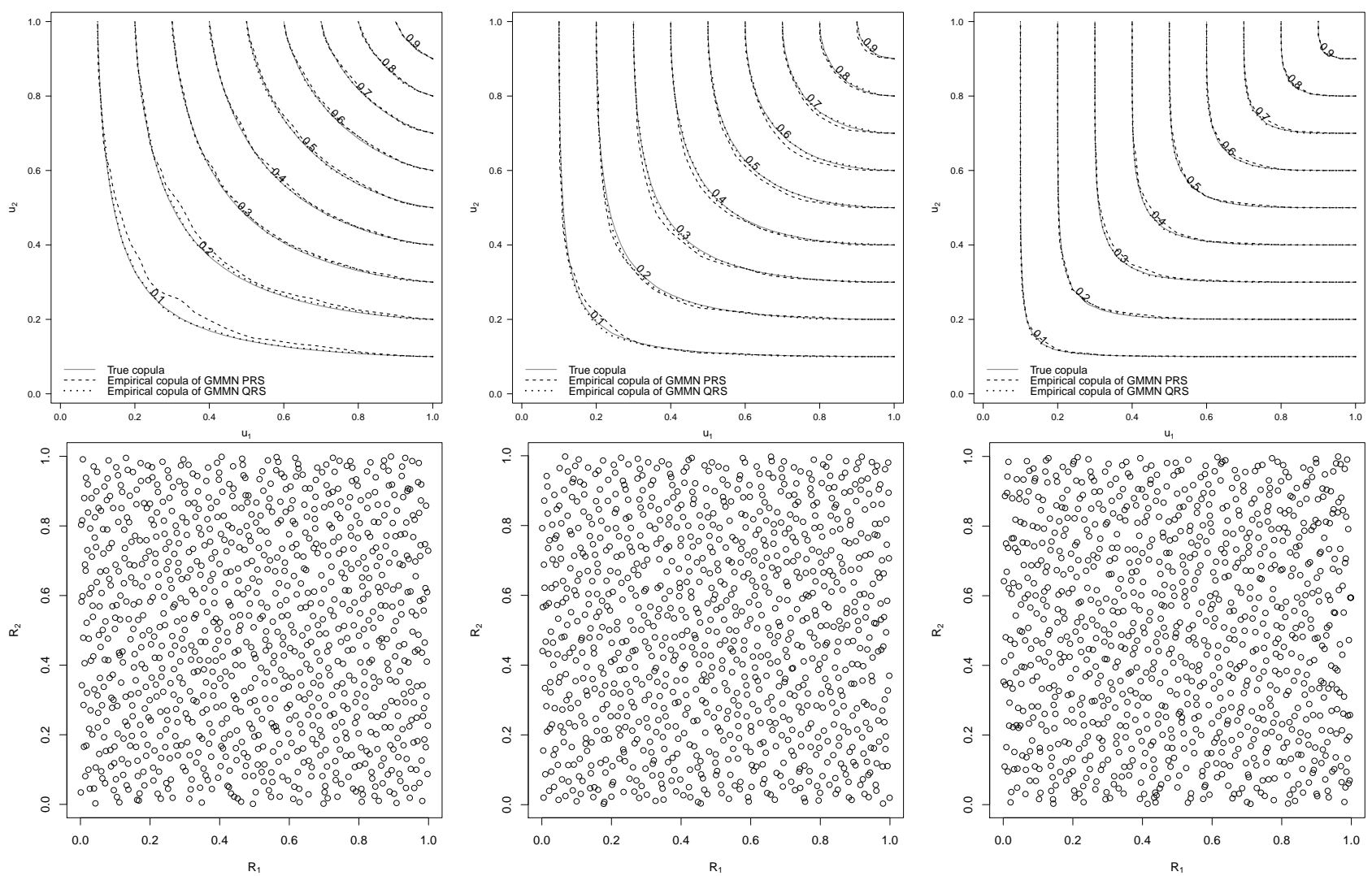

Figure 3 Top row contains contour plots of true Gumbel copulas with $\tau=0.25$ (left), 0.50 (middle) and 0.75 (right) along with the corresponding contour plots of empirical copulas based on GMMN PRS and GMMN QRS, both of size $n_{\text {gen }}=1000$. Bottom row contains Rosenblatttransformed GMMN QRS corresponding to the same three Gumbel copulas. 
3 GMMN pseudo- and quasi-random samples for copula models
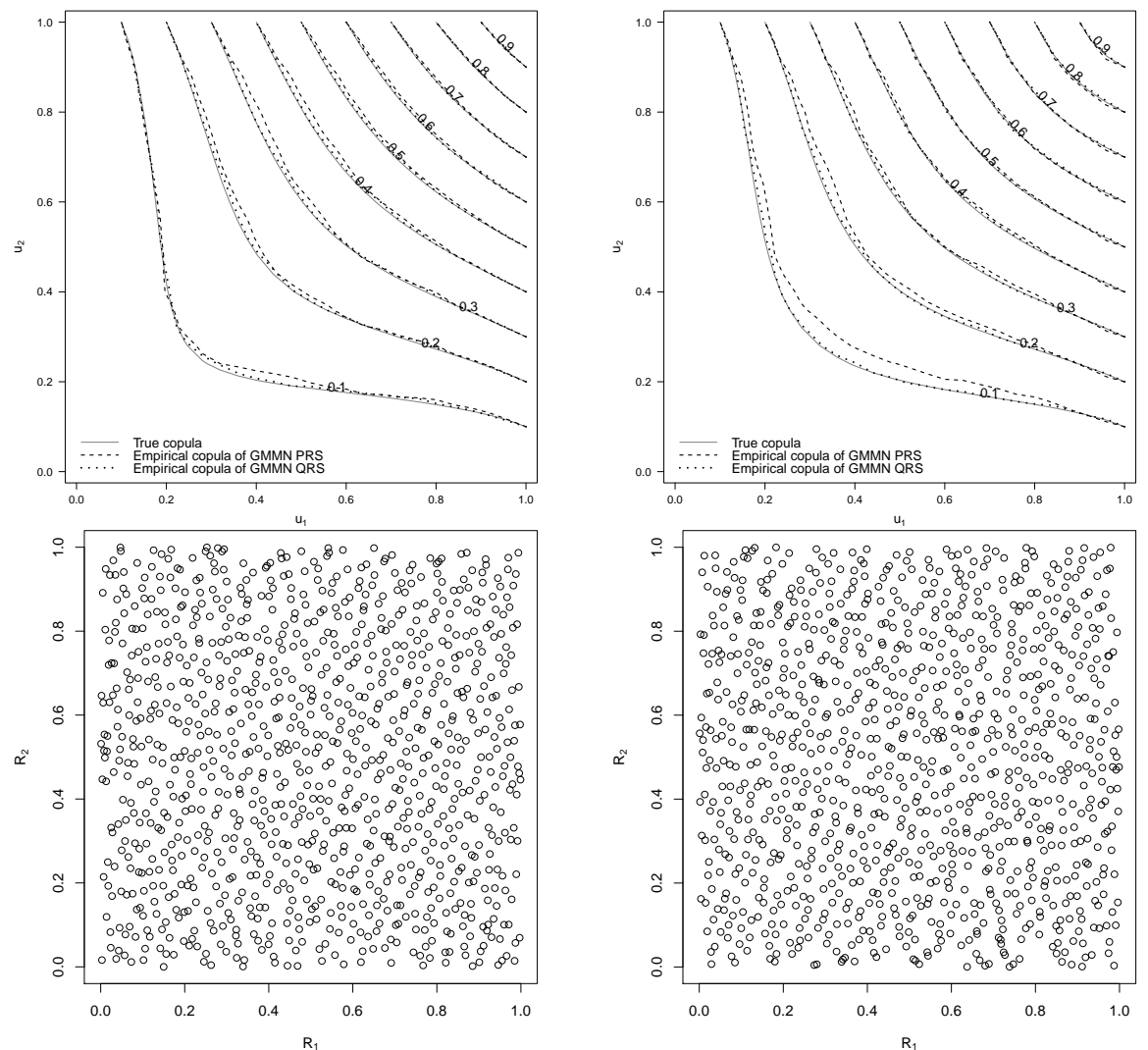

Figure 4 Top row contains contour plots of true Clayton- $t(90)$ (left) and Gumbel-t(90) (right) mixture copulas along with the corresponding contour plots of empirical copulas based on GMMN PRS and GMMN QRS, both of size $n_{\text {gen }}=1000$. Bottom row contains Rosenblatt-transformed GMMN QRS corresponding to the same two mixture copulas. 
Bivariate Marshall-Olkin copulas are of the form

$$
C\left(u_{1}, u_{2}\right)=\min \left\{u_{1}^{1-\alpha_{1}} u_{2}, u_{1} u_{2}^{1-\alpha_{2}}\right\}, \quad u_{1}, u_{2} \in[0,1],
$$

where $\alpha_{1}, \alpha_{2} \in[0,1]$. A notable feature of Marshall-Olkin copulas is that they have both an absolutely continuous component and a singular component. In particular, the singular component is determined by all points which satisfy $u_{1}^{\alpha_{1}}=u_{2}^{\alpha_{2}}$. Accurately capturing this singular component may present a different challenge for GMMNs, which is why we included this copula despite the fact that there also exists a CDM for this copula; see Cambou et al. (2017). As an example for visual assessment, we consider a Marshall-Olkin copula with $\alpha_{1}=0.75$ and $\alpha_{2}=0.60$.

We also consider three mixture models, all of which are equally weighted two-component mixture copulas with one component being a 90-degree-rotated $t_{4}$ copula with $\tau=0.50$. The first two models are the Clayton- $t(90)$ and Gumbel- $t(90)$ mixture copulas as previously introduced. The second component in the third model is a Marshall-Olkin copula with parameters $\alpha_{1}=0.75$ and $\alpha_{2}=0.60$. We refer to this third model as the MO-t(90) copula.

Figures 57 display pseudo-random samples (left column) from a $(2,1)$-nested Clayton copula as in (10), a MO copula, and the three mixture copulas, respectively, along with GMMN pseudo-random samples (middle column) and GMMN quasi-random samples (right column) corresponding to each copula $C$. The similarity between the GMMN pseudo-random samples in the middle column and the pseudo-random samples in the left column indicate that the copulas $C$ were learned sufficiently well by their corresponding GMMNs. Note that in the case of the nested Clayton copula example, we can only comment on how well the bivariate margins of the copula $C$ were learned. From the right columns, we can mainly observe that the GMMN quasi-random samples contain less gaps and clusters when compared with the corresponding pseudo-random and GMMN pseudo-random samples. The fact that GMMNs were capable of learning the main features, including the singular components, of the MO copula and the MO-t(90) mixture copula is particularly noteworthy given how challenging it seems to be to learn a Lebesgue null set from a finite amount of samples.
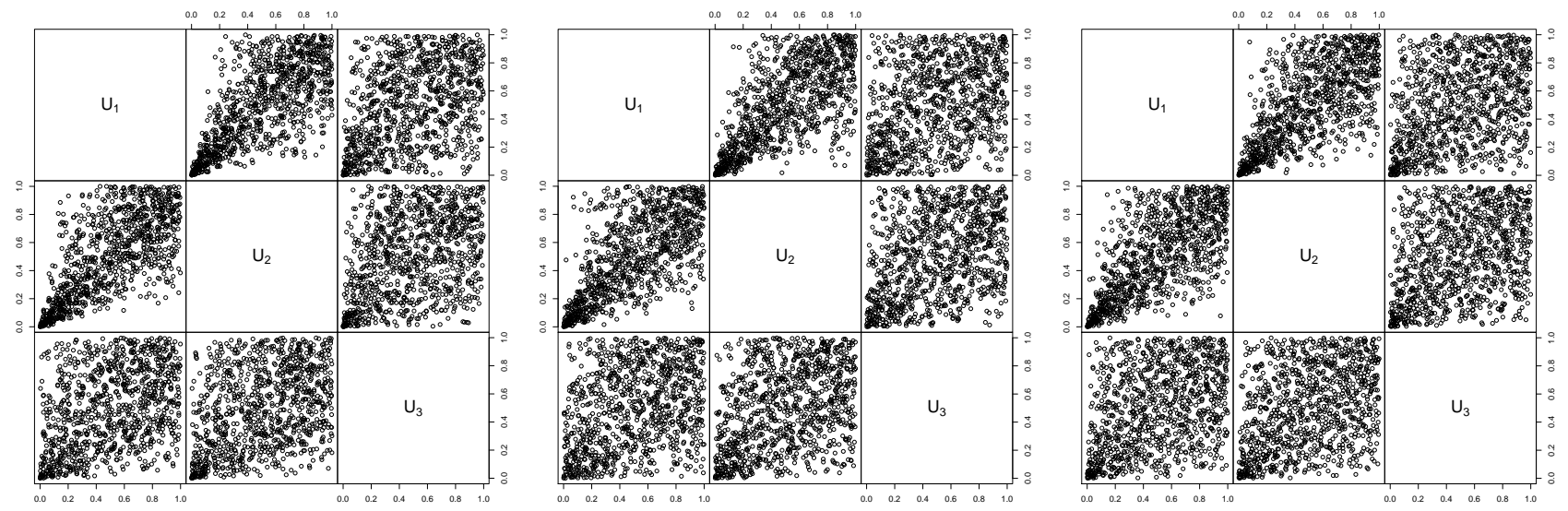

Figure 5 Pseudo-random samples (PRS; left), GMMN pseudo-random samples (GMMN PRS; middle) and GMMN quasi-random samples (GMMN QRS; right), all of size $n_{\text {gen }}=1000$, from a $(2,1)$-nested Clayton copula as in 10$)$ with $\tau_{0}=0.25$ and $\tau_{1}=0.50$.

\subsection{Assessment of GMMN samples by the Cramér-von Mises statistic}

After a purely visual inspection of the generated samples, we now assess the quality of GMMN pseudo-random and GMMN quasi-random samples more formally with the help of a goodness-of-fit 

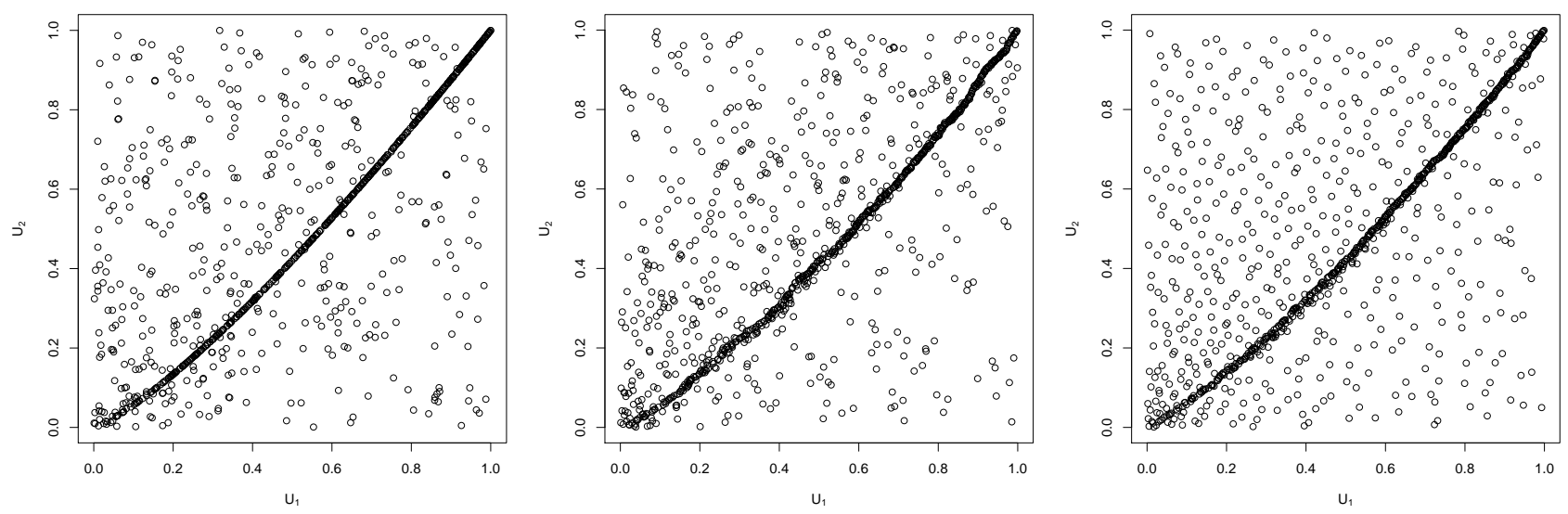

Figure 6 PRS (left), GMMN PRS (middle) and GMMN QRS (right), all of size $n_{\text {gen }}=1000$, from a Marshall-Olkin copula with $\alpha_{1}=0.75$ and $\alpha_{2}=0.60$ (Kendall's tau equals 0.5 ).

statistic. Since bivariate copulas have been investigated in detail in the previous section, we focus on higher-dimensional copulas in this section.

Specifically, we use the Cramér-von Mises statistic (Genest, Rémillard, et al., 2009),

$$
S_{n_{\mathrm{gen}}}=\int_{[0,1]^{d}} n_{\mathrm{gen}}\left(C_{n_{\mathrm{gen}}}(\boldsymbol{u})-C(\boldsymbol{u})\right)^{2} \mathrm{~d} C_{n_{\mathrm{gen}}}(\boldsymbol{u})
$$

where the empirical copula

$$
C_{n_{\text {gen }}}(\boldsymbol{u})=\frac{1}{n_{\text {gen }}} \sum_{i=1}^{n_{\text {gen }}} \mathbb{1}\left\{\hat{U}_{i 1} \leq u_{1}, \ldots, \hat{U}_{i d} \leq u_{d}\right\}, \quad \boldsymbol{u} \in[0,1]^{d},
$$

is the empirical distribution function of the pseudo-observations. For $n_{\text {gen }}=1000$ and each copula $C$, we compute $B=100$ realizations of $S_{n_{\text {gen }}}$ three times - once for the case where $\hat{\boldsymbol{U}}_{i}, i=1, \ldots, n_{\text {gen }}$, are pseudo-observations of the true underlying copula (as benchmark), once for GMMN pseudo-random samples and once for GMMN quasi-random samples. We then use box plots to depict the distribution of $S_{n_{\text {gen }}}$ in each case. Figure 8 displays these box plots for $t_{4}$ (top row), Clayton (middle row) and Gumbel (bottom row) copulas of dimensions $d=5$ (left column), $d=10$ (right column) and $\tau=0.50$. Similarly, Figure 9 displays such box plots for $d$-dimensional nested Clayton (left column) and nested Gumbel (right column) copulas for $d=3$ (top row), $d=5$ (middle row) and $d=10$ (bottom row). The three-dimensional NACs have a structure as given by 10 with $\tau_{0}=0.25$ and $\tau_{1}=0.50$; the fivedimensional NACs have structure $C_{0}\left(C_{1}\left(u_{1}, u_{2}\right), C_{2}\left(u_{3}, u_{4}, u_{5}\right)\right)$ with corresponding $\tau_{0}=0.25, \tau_{1}=0.50$ and $\tau_{2}=0.75$; and the ten-dimensional NACs have structure $C_{0}\left(C_{1}\left(u_{1}, \ldots, u_{5}\right), C_{2}\left(u_{6}, \ldots, u_{10}\right)\right)$ with corresponding $\tau_{0}=0.25, \tau_{1}=0.50$ and $\tau_{2}=0.75$.

We can observe from both figures that the distributions of $S_{n_{\text {gen }}}$ for pseudo-random samples from $C$ and from the GMMN are similar, with slightly higher $S_{n_{\text {gen }}}$ values for the GMMN pseudo-random samples, especially for $d=10$. Additionally, we can observe that the distribution of $S_{n_{\text {gen }}}$ based on the GMMN quasi-random samples is closer to zero than that of the GMMN pseudo-random samples. This provides some evidence that the low-discrepancy of input RQMC points sets has been preserved under the respective (trained) GMMN transform.

We also see that $S_{n_{\text {gen }}}$ values based on the GMMN quasi-random samples are clearly lower than $S_{n_{\text {gen }}}$ values based on the copula pseudo-random samples, with the exception of (some) copulas for $d=10$ where the distributions of $S_{n_{\mathrm{gen}}}$ are more similar. 

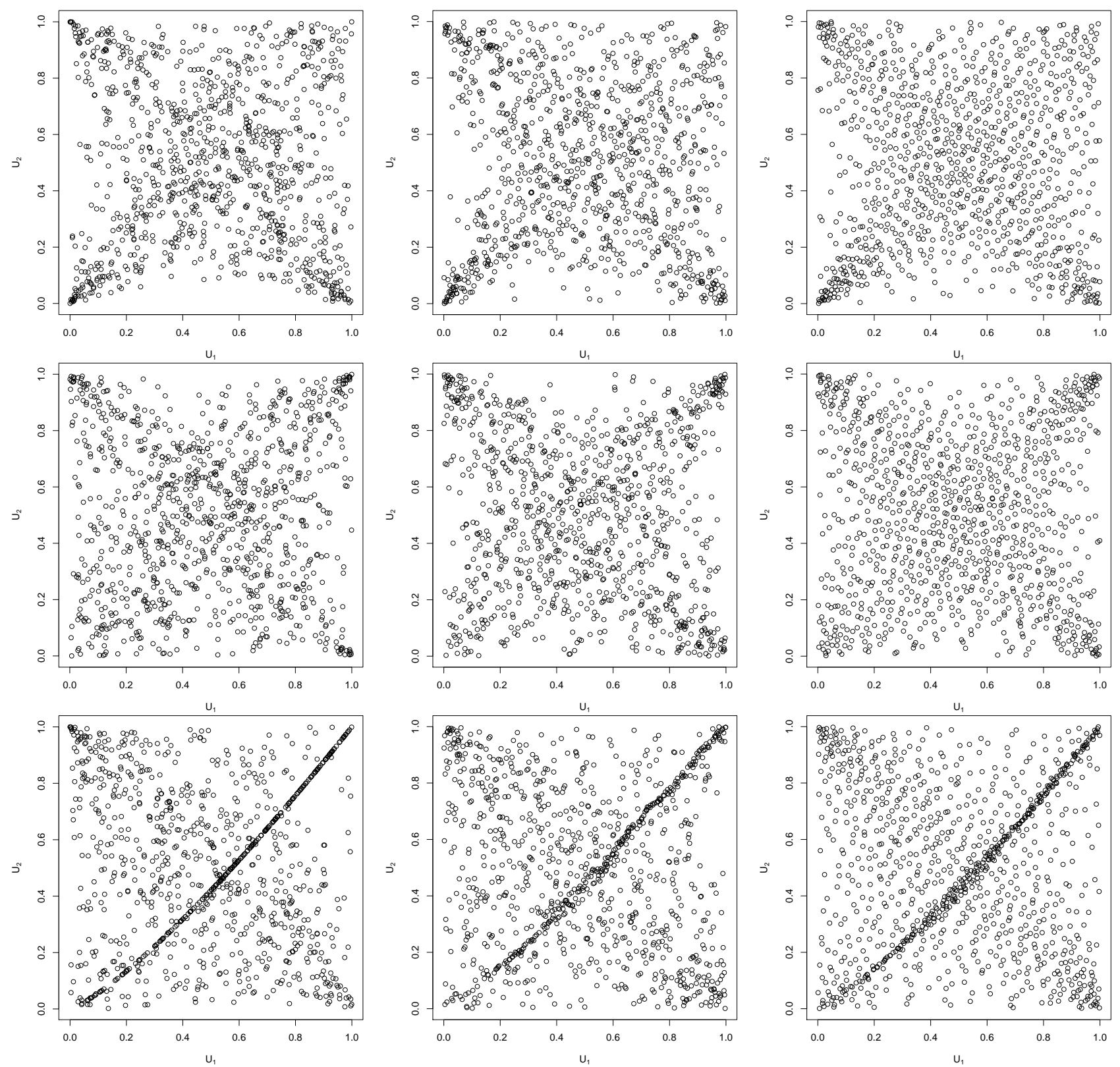

Figure 7 PRS (left column), GMMN PRS (middle column) and GMMN QRS (right column), all of size $n_{\text {gen }}=1000$, from a Clayton- $t(90)$ (top row), Gumbel- $t(90)$ (middle row) and a MO- $t(90)$ mixture (bottom row) copula. 
3 GMMN pseudo- and quasi-random samples for copula models
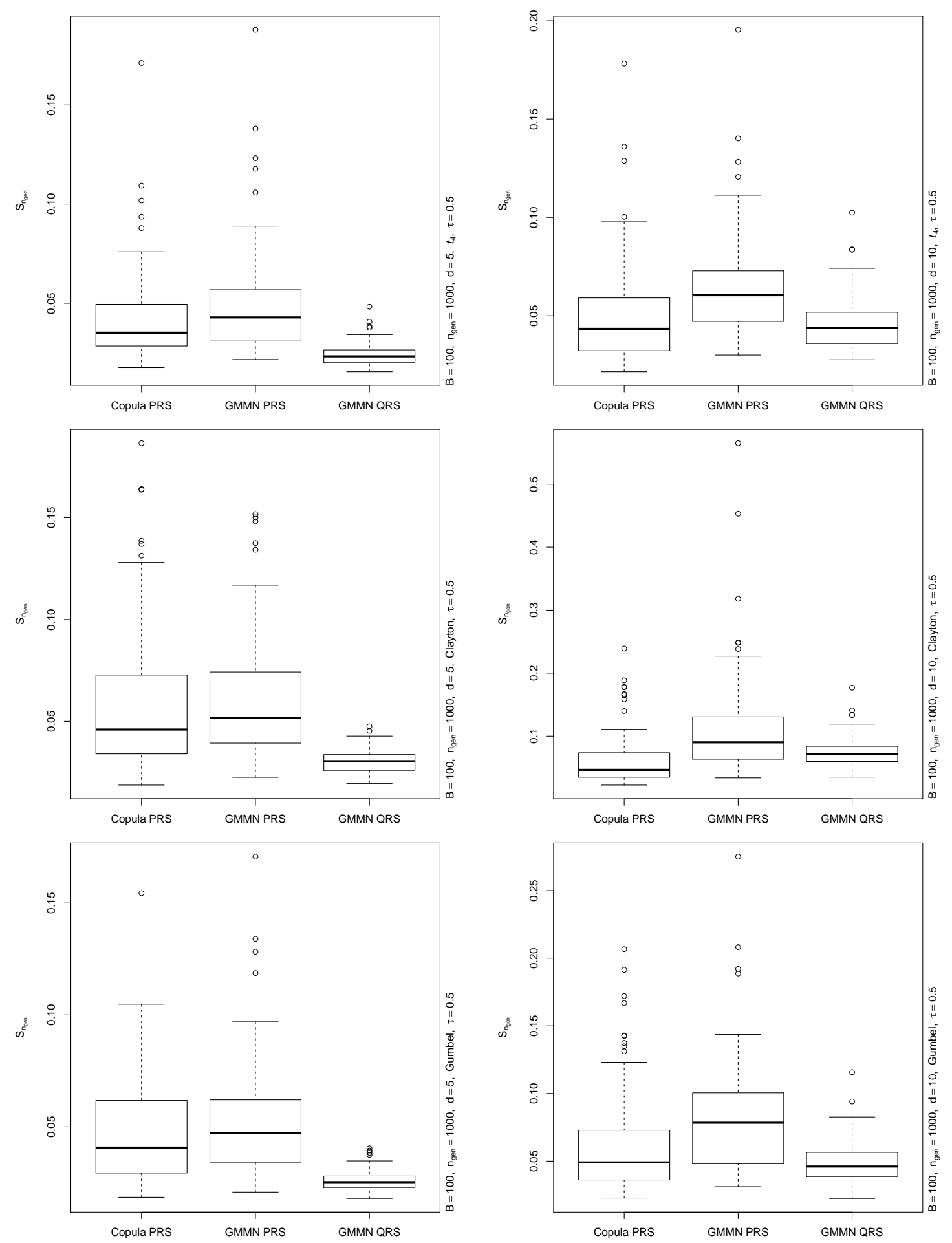

Figure 8 Box plots based on $B=100$ realization of $S_{n_{\text {gen }}}$ computed from (i) a pseudo-random sample (PRS) of $C$ (denoted by Copula PRS), (ii) a GMMN pseudo-random sample (denoted by GMMN PRS) and (iii) a GMMN quasi-random sample (denoted by GMMN QRS) — all of size $n_{\text {gen }}=1000$ - for a $t_{4}$ (top row), Clayton (middle row) and Gumbel (bottom row) copula with $\tau=0.5$ as well as $d=5$ (left column) and $d=10$ (right column). 
3 GMMN pseudo- and quasi-random samples for copula models
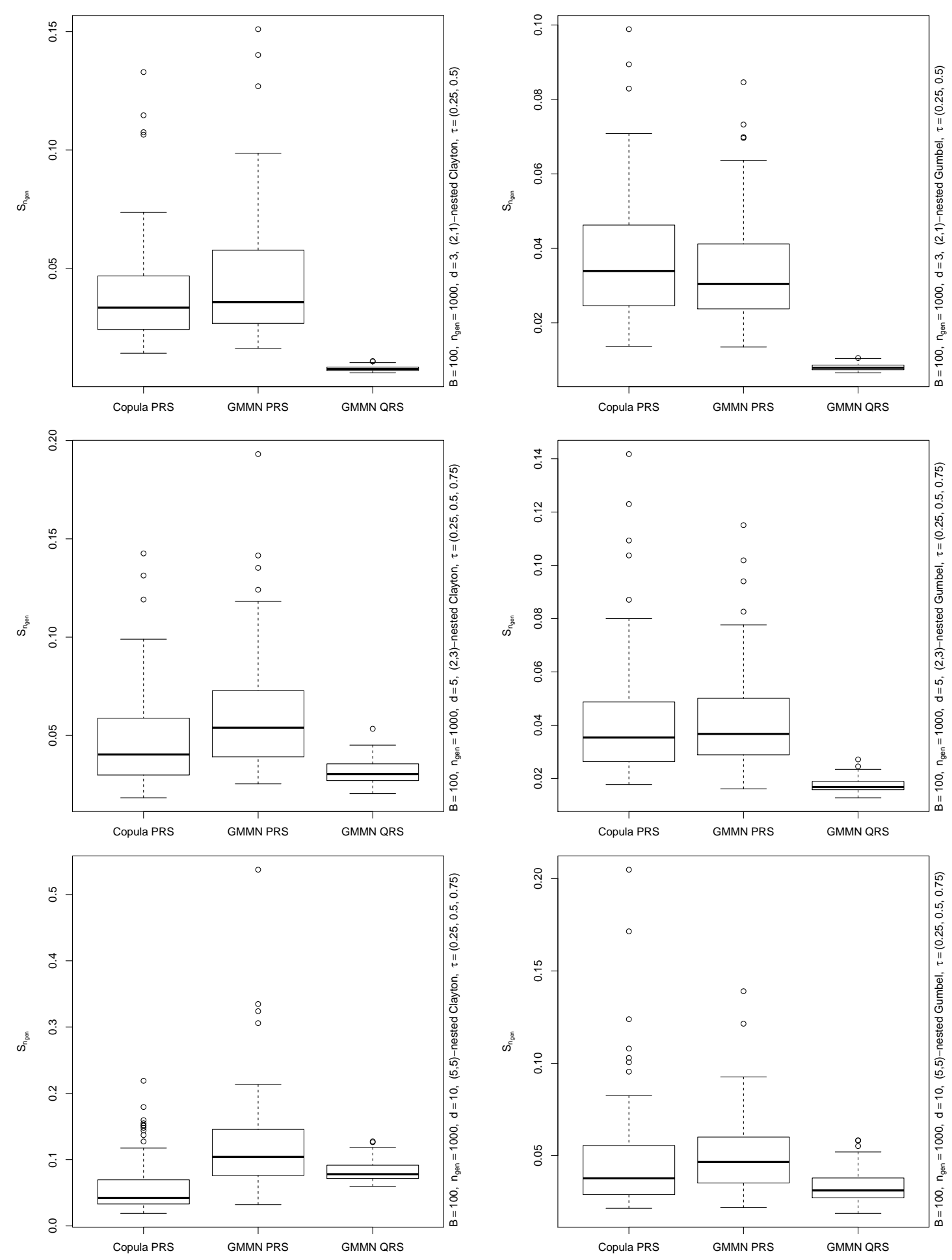

Figure 9 As Figure 8 but for nested Clayton (left column) and nested Gumbel (right column) copulas and for $d=3$ (top row), $d=5$ (middle row) and $d=10$ (bottom row). 


\section{Convergence analysis of the RQMC estimator}

In this section we numerically investigate the variance-reduction properties of the GMMN RQMC estimator $\hat{\mu}_{n_{\text {gen }}}^{\mathrm{NN}}$ in $(7)$ for two functions $\Psi$ and transforms $q=f_{\hat{\boldsymbol{\theta}}} \circ \Phi^{-1}$ corresponding to different copulas $C$. We compare $\hat{\mu}_{n_{\text {gen }}}^{\mathrm{NN}}$ with estimators based on standard copula pseudo-random and, where available, copula quasi-random samples. For the latter, we follow Cambou et al. (2017) but note that quasi-random sampling procedures are only available for some of the copulas we consider here; for others, the procedures are either too slow (e.g., for Gumbel copulas; see Appendix B) or not known at all (e.g., for nested Clayton or Gumbel copulas).

We consider two different types of functions $\Psi$. The first is a test function primarily used in the QMC literature to test the performance of $\hat{\mu}_{n_{\text {gen }}}^{\mathrm{NN}}$ in terms of its ability to preserve the low-discrepancy of $\tilde{P}_{n_{\text {gen }}}$. The second function $\Psi$ is motivated from a practical application in risk management. For both functions, standard deviation estimates will be computed to compare convergence rates, based on $B=25$ randomized point sets $\tilde{P}_{n_{\text {gen }}}$ for each of $n_{\text {gen }} \in\left\{2^{10}, 2^{10.5}, \ldots, 2^{18}\right\}$ to help roughly gauge the convergence rate for all estimators. Furthermore, regression coefficients $\alpha$ (obtained by regressing the logarithm of the standard deviation on the logarithm of $n_{\text {gen }}$ ) are computed and displayed to allow for an easy comparison of the corresponding convergence rates $O\left(n_{\mathrm{gen}}^{-\alpha}\right)$ with the theoretical convergence rate $O\left(n_{\text {gen }}^{-0.5}\right)$ of the Monte Carlo estimator's standard deviation. For RQMC estimators one can expect $\alpha$ to be larger than 0.5 , but with an upper bound of $1.5-\varepsilon$, where $\varepsilon$ increases with dimension $d$; see Theorem A.3 for further details.

\subsection{A test function}

The test function we consider is the Sobol' $g$ function (Faure and Lemieux, 2009) based on the Rosenblatt transform and is given by

$$
\Psi_{1}(\boldsymbol{U})=\prod_{j=1}^{d} \frac{\left|4 R_{j}-2\right|+j}{1+j},
$$

where $R_{1}=U_{1}$ and, for $j=2, \ldots, d$ and if $\boldsymbol{U} \sim C, R_{j}=C_{j \mid 1, \ldots, j-1}\left(U_{j} \mid U_{j-1}, \ldots, U_{1}\right)$ denotes the conditional distribution function of $U_{j}$ given $U_{1}, \ldots, U_{j-1}$.

Figure 10 shows plots of standard deviation estimates for estimating $\mathbb{E}\left(\Psi_{1}(\boldsymbol{U})\right.$ ) for $t_{4}$ copulas (top row), Clayton (middle row) and Gumbel (bottom row) copulas in dimensions $d=2$ (left column), $d=5$ (middle column) and $d=10$ (right column). For the $t_{4}$ and Clayton copulas we numerically compare the efficiency of the GMMN RQMC estimator (with legend label "GMMN QRS") with the copula RQMC estimator based on the CDM method (with legend label "Copula QRS") and the MC estimator (with legend label "Copula PRS"). For the Gumbel copula, however, the CDM approach ("Copula QRS") is computationally not feasible; see Section 3.2.1 and Appendix B. The legend of each plot also provides the regression coefficient $\alpha$ which indicates the convergence rate of each estimator.

From Figure 10, we observe that the GMMN RQMC estimator clearly outperforms the MC estimator. Naturally, so does the copula RQMC estimator for the copulas for which it is available. On the one hand, the rate of convergence of the GMMN RQMC estimator reduces with increasing copula dimensions; see also the decreasing regression coefficients $\alpha$ when moving from the two- to the ten-dimensional case. On the other hand, the GMMN RQMC estimator still outperforms the MC estimator. 

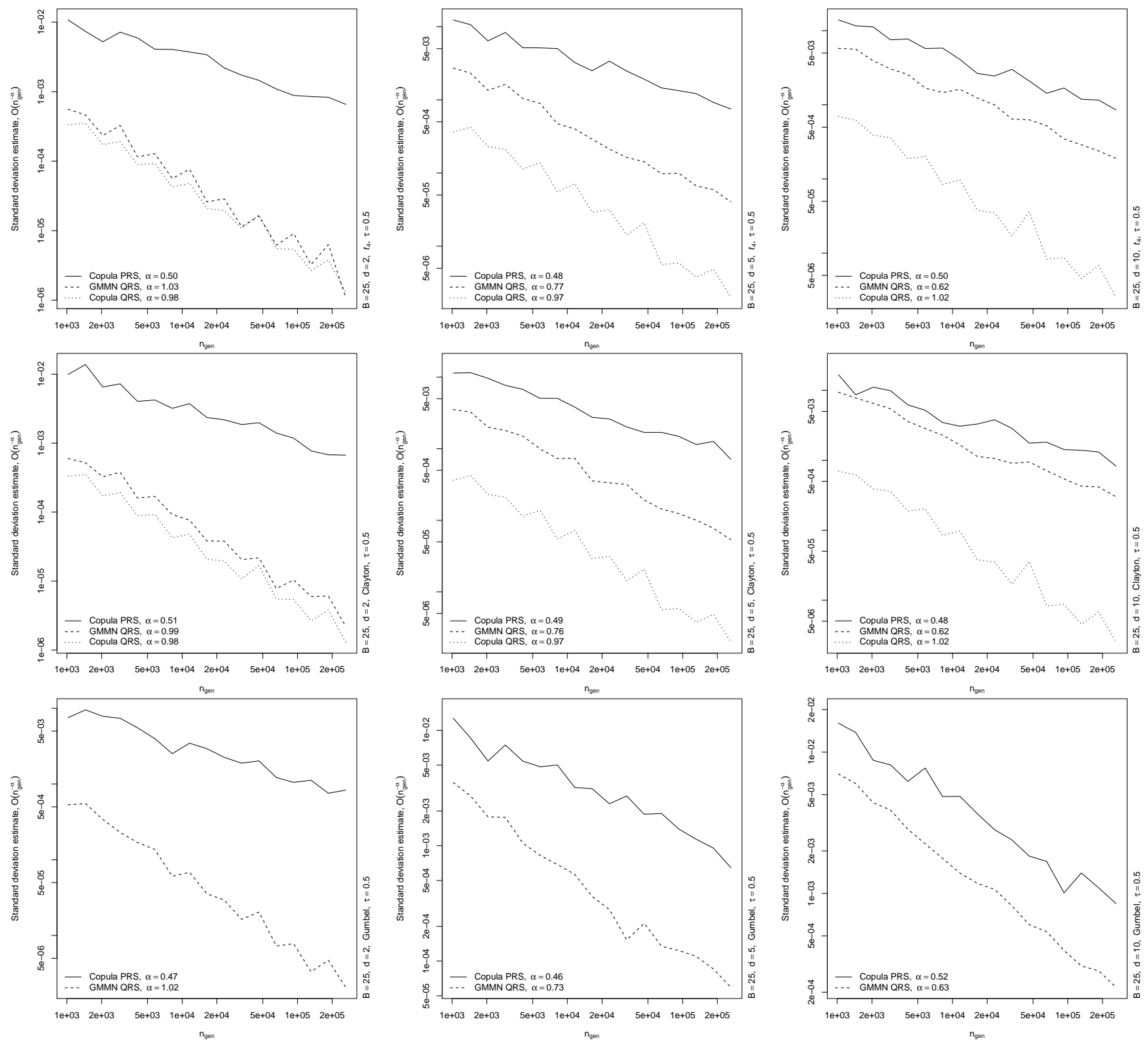

Figure 10 Standard deviation estimates based on $B=25$ replications for estimating $\mathbb{E}\left(\Psi_{1}(\boldsymbol{U})\right)$, expectation of the Sobol' $\mathrm{g}$ function, via $\mathrm{MC}$ based on a pseudo-random sample (PRS), via the copula RQMC estimator (whenever available; rows 1-2 only) and via the GMMN RQMC estimator. Note that in rows $1-3, d \in\{2,5,10\}$. 


\section{A real-data example}

\subsection{An example from risk management practice}

Consider modeling the dependence of $d$ risk-factor changes (for example, logarithmic returns) of a portfolio; see McNeil et al. (2015, Chapters 2,6 and 7). We now demonstrate the efficiency of our GMMN RQMC estimator by considering the expected shortfall of the aggregate loss, a popular risk measure in quantitative risk management practice.

Specifically, if $\boldsymbol{X}=\left(X_{1}, \ldots, X_{d}\right)$ denotes a random vector of risk-factor changes with $\mathrm{N}(0,1)$ margins, the aggregate loss is $S=\sum_{j=1}^{d} X_{j}$. The expected shortfall $\mathrm{ES}_{0.99}$ at level 0.99 of $S$ is given by

$$
\operatorname{ES}_{0.99}(S)=\frac{1}{1-0.99} \int_{0.99}^{1} F_{S}^{-1}(u) \mathrm{d} u=\mathbb{E}\left(S \mid S>F_{S}^{-1}(0.99)\right)=\mathbb{E}\left(\Psi_{2}(\boldsymbol{X})\right)
$$

where $F_{S}^{-1}$ denotes the quantile function of $S$. As done previously, various copulas will be used to model the dependence between the components of $\boldsymbol{X}$.

Figure 11 shows plots of standard deviation estimates for estimating $\mathbb{E}\left(\Psi_{2}(\boldsymbol{X})\right)$. The first three rows contain results for the same copula models as considered in Section 4.1. The fourth row contains results for nested Gumbel copula models with dimension $d=3$ (left column), $d=5$ (middle column) and $d=10$ (right column). The specific hierarchical structures and parameterization have been described earlier in Section 3.3 note that there is no quasi-random sampling procedure known for these copulas. We can observe from the plots that the GMMN RQMC estimator outperforms the MC estimator. Similar as before, we see a decrease in the convergence rate of the GMMN RQMC estimator as the copula dimension increases, although it still outperforms the MC estimator.

\section{A real-data example}

In this section, we present examples based on real-data to show how our method can be useful in practice. To this end, we consider applications from finance and risk management. Such applications often involve the modeling of dependent multivariate return data in order to estimate various quantities $\mu$ of interest. In this context, utilizing GMMNs for dependence modeling can yield two key advantages. Firstly, GMMNs are highly flexible and hence can model dependence structures not adequately captured by prominent parametric copula models; see, e.g., Hofert and Oldford (2018) for the latter point. Secondly, as demonstrated in Sections 3 and 4 , one can readily generate GMMN quasi-random samples to achieve variance reduction when estimating $\mu$; this is especially advantageous as oftentimes oversimplified parametric models are chosen just so that this can be achieved. In this section, we model asset portfolios consisting of S\&P 500 constituents to showcase these advantages. All results are reproducible with the demo GMMN_QMC_data of the $\mathrm{R}$ package gnn.

\subsection{Portfolios of S\&P 500 constituents}

We consider 10 constituent time series from the S\&P 500 in the time period from 1995-01-01 to 2015-12-31. The selected constituents include three stocks from the information technology sector Intel Corp. (INTC), Oracle Corp. (ORCL) and International Business Machines Corp. (IBM); three stocks from the financial sector - Capital One Financial Corp. (COF), JPMorgan Chase \& Co. (JPM) and American International Group Inc (AIG); and four stocks from the industrial sector - 3M Company (MMM), Boeing Company (BA), General Electric (GE) and Caterpillar Inc. (CAT). We also investigate sub-portfolios of stocks with dimensions $d=5$ (consisting of INTC, ORCL, IBM, COF and $\mathrm{AIG}$ ) and $d=3$ (consisting of INTC, IBM and AIG). The data are obtained from the R package qrmdata. 
5 A real-data example
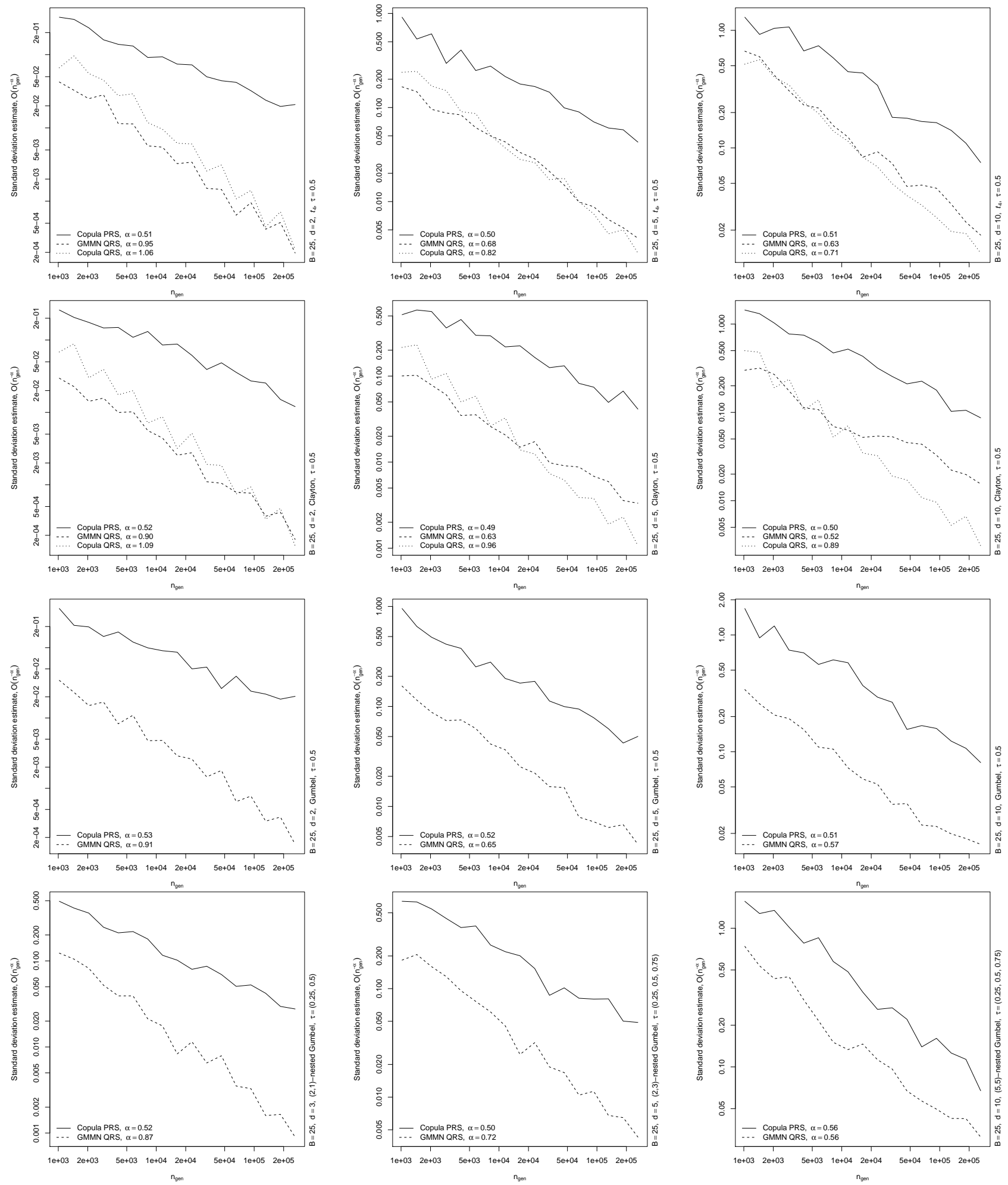

Figure 11 Standard deviation estimates based on $B=25$ replications for estimating $\mathbb{E}\left(\Psi_{2}(\boldsymbol{X})\right)$, the expected shortfall $\mathrm{ES}_{0.99}(S)$, via $\mathrm{MC}$ based on a PRS, via the copula RQMC (whenever available; rows 1-2 only) and via the GMMN RQMC estimator. Note that in rows 1-3, $d \in\{2,5,10\}$, whereas in row $4, d \in\{3,5,10\}$. 


\section{A real-data example}

To account for marginal temporal dependencies, we follow the copula-GARCH approach (Jondeau and Rockinger, 2006 Patton, 2006) and model each marginal time series of log-returns by a $\operatorname{ARMA}(1,1)-$ GARCH $(1,1)$ model with standardized $t$ innovation distributions (deGARCHing). We then extract the marginal standardized residuals (i.e., the realizations of the standardized $t$ innovations) and compute, for each of the three portfolios, their pseudo-observations for the purpose of modeling the cross-sectional dependence among the corresponding portfolio's log-return series.

\subsection{Assessing the fit of dependence models}

As models for the pseudo-observations of each of the three portfolios we use prominent parametric copulas (Gumbel, Clayton, exchangeable normal, unstructured normal, exchangeable $t$ and unstructured $t$ ) and GMMNs of the same architecture and with the same training setup as detailed in Section 3.1 . The rather small number of training data points $\left(n_{\operatorname{trn}}=5287\right)$ allows us to use $n_{\text {bat }}=n_{\text {trn }}$ here and hence directly train with the entire dataset. All parametric copulas are fitted using the maximum pseudo-likelihood method; see Hofert, Kojadinovic, et al. (2018, Section 4.1.2).

To evaluate the fit of a dependence model, we use a Cramér-von-Mises type test statistic introduced by Rémillard and Scaillet $(2009)$ to assess the equality of two empirical copulas. This statistic is defined as

$$
S_{n_{\mathrm{trn}}, n_{\mathrm{gen}}}=\int_{[0,1]^{d}}\left(\sqrt{\frac{1}{n_{\mathrm{gen}}}+\frac{1}{n_{\mathrm{trn}}}}\right)^{-1}\left(C_{n_{\mathrm{gen}}}(\boldsymbol{u})-C_{n_{\mathrm{trn}}}(\boldsymbol{u})\right)^{2} \mathrm{~d} \boldsymbol{u},
$$

where $C_{n_{\text {gen }}}(\boldsymbol{u})$ and $C_{n_{\text {trn }}}(\boldsymbol{u})$ are the empirical copulas, defined according to (11), of the $n_{\text {gen }}$ samples generated from the fitted dependence model and the $n_{\text {trn }}$ pseudo-observations used to fit the dependence model, respectively. For how $S_{n_{\mathrm{trn}}, n_{\mathrm{gen}}}$ is evaluated, see Rémillard and Scaillet (2009, Section 2).

For each of the three portfolios and each of the seven dependence models considered, we compute $B$ realizations of $S_{n_{\text {trn }}, n_{\text {gen }}}$ based on $n_{\text {gen }}=10000$ pseudo-random samples generated from the fitted dependence model and the $n_{\text {trn }}=5287$ pseudo-observations of each portfolio considered. Figure 12 displays box plots depicting the distribution of $S_{n_{\mathrm{trn}}, n_{\mathrm{gen}}}$ for each portfolio and dependence model. Across all three portfolios, we can observe that the distribution of $S_{n_{\text {trn }}, n_{\text {gen }}}$ based on GMMN models is concentrated closer to zero than those of each fitted parametric copula. In fact, the difference in distributions of $S_{n_{\text {trn }}, n_{\text {gen }}}$ realizations between GMMN models and the best parametric copula model (a $t$-copula with unstructured correlation matrix) is most noticeable for $d=10$, where an adequate fit becomes more challenging for these parametric copulas. For each of the three portfolios, a GMMN provides the best fit. Hence, we use these fitted GMMNs to model the underlying dependence structure for the three portfolios in each of three applications considered next.

\subsection{Assessing the variance reduction effect}

In three applications we study the variance reduction effect of our GMMN RQMC estimator $\hat{\mu}_{n_{\text {gen }}}^{\mathrm{NN}}$ computed from quasi-random samples in comparison to the GMMN MC estimator $\hat{\mu}_{n_{\text {gen }}}^{\mathrm{NN}, \mathrm{MC}}$ computed from pseudo-random samples.

Our first application concerns the estimation of the expected shortfall $\mu=\operatorname{ES}_{0.99}(S)$ for $S=\sum_{j=1}^{d} X_{j}$ as in Section 4.2, where the margins of $\boldsymbol{X}=\left(X_{1}, \ldots, X_{d}\right)$ are now the fitted standardized $t$ distributions as obtained by deGARCHing and the dependence structure is the previously fitted GMMN. This is a classical task in risk management practice according to the Basel guidelines. As a second application we consider a capital allocation problem which concerns estimating how to allocate an amount of risk capital (e.g., computed as $\mathrm{ES}_{0.99}(S)$ ) to each of $d$ business lines. Without loss of 


\section{A real-data example}
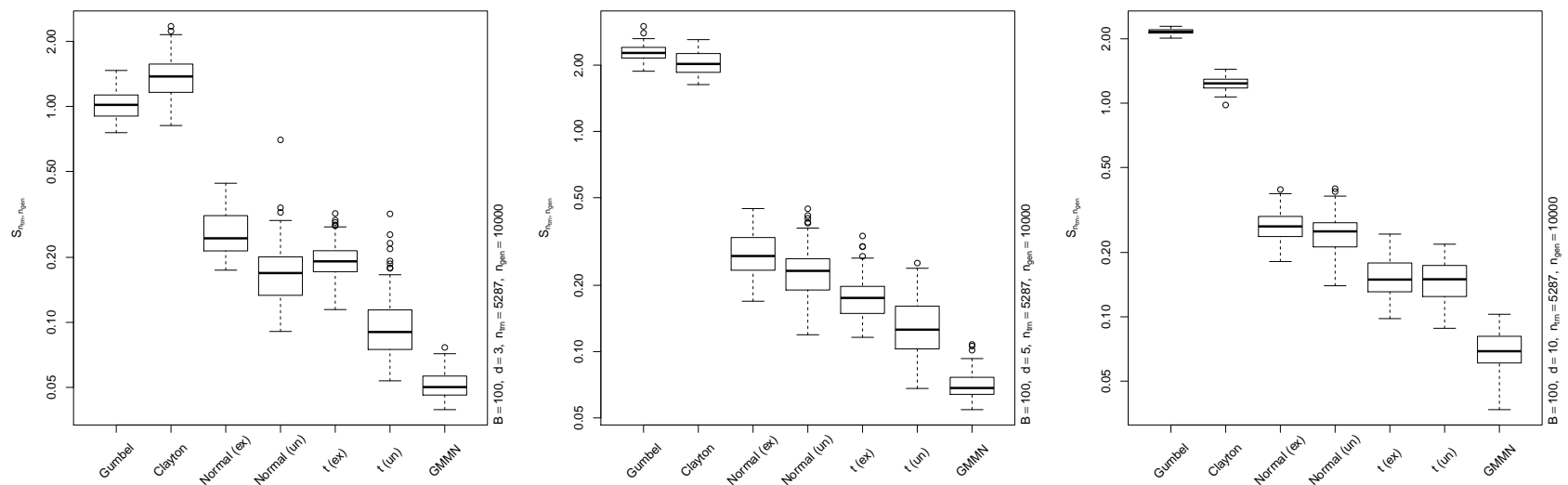

Figure 12 Box plots based on $B=100$ realizations of $S_{n_{\operatorname{trn}}, n_{\operatorname{gen}}}$ computed for portfolios of dimensions $d=3$ (left), $d=5$ (middle) and $d=10$ (right) and for each fitted dependence model using a pseudo-random sample of size $n_{\text {gen }}=10000$ from each corresponding fitted model.

generality, we consider one business line, the first, and estimate the expected shortfall contribution $\mu=\mathrm{AC}_{1,0.99}=\mathbb{E}\left(X_{1} \mid S>F_{S}^{-1}(0.99)\right)$ according to the Euler principle; see McNeil et al. 2015 , Section 8.5). Our third application comes from finance and concerns the estimation of the expected payoff $\mu=\mathbb{E}\left(\exp (-r(T-t)) \max \left\{\left(\sum_{j=1}^{d} S_{T, j}\right)-K, 0\right\}\right)$ of a European basked call option, where $r$ denotes the continuously compounded annual risk-free interest rate, $t$ denotes the current time point, $T$ the maturity in years and $K$ the strike price. We assume a Black-Scholes framework for the marginal stock prices $\left(S_{T, 1}, \ldots, S_{T, d}\right)$ at maturity $T$, so $S_{T, j} \sim \operatorname{LN}\left(\log \left(S_{t, j}\right)+\left(r-\sigma_{j}^{2} / 2\right)(T-t), \sigma_{j}^{2}(T-t)\right)$, where $S_{t, j}$ denotes the last available stock price of the $j$ th constituent (i.e., the close price on 2015-12-31) and $\sigma_{j}$ denotes the volatility of the $j$ th constituent (estimated by the standard deviation of its log-returns over the time period from 2014-01-01 to 2015-12-31). The dependence structure of $\left(S_{T, 1}, \ldots, S_{T, d}\right)$ is modeled by the previously fitted GMMN. Furthermore, we chose $t=0$ to be the last available point in the data period considered (i.e., 2015-12-31), $T=1$ and $r=0.01$. The strike prices $K$ were chosen about $0.5 \%$ higher than the average stock price of all stocks in the respective portfolio at $t=0$.

For each of the three portfolios and for each of the three expectations $\mu$ considered, we compute $B=200$ realizations of the GMMN MC estimator $\hat{\mu}_{n_{\text {gen }}^{N N}}^{\mathrm{NN} \text { MC }}$ and the GMMN RQMC estimator $\hat{\mu}_{n_{\text {gen }}}^{\mathrm{NN}}$, using $n_{\text {gen }}=10^{5}$ samples for both estimators. Figure 13 displays box plots of these realizations of $\hat{\mu}_{n_{\text {gen }}}^{\mathrm{NN}, M C}$ (with x-axis label "GMMN PRS") and of $\hat{\mu}_{n_{\text {gen }}}^{\mathrm{NN}}$ (with x-axis label "GMMN QRS") for $\mathrm{ES}_{0.99}$ (left column), $\mathrm{AC}_{1,0.99}$ (middle column), the expected payoff of the basket call option (right column) and for the portfolio in dimension $d=3$ (top row), $d=5$ (middle row) and $d=10$ (bottom row). Additionally, to quantify the variance reduction effect of $\hat{\mu}_{n_{\text {gen }}}^{\mathrm{NN}}$ over $\hat{\mu}_{n_{\mathrm{gen}}}^{\mathrm{NN}, \mathrm{MC}}$, we report in the secondary $\mathrm{y}$-axis of each box plot the estimated variance reduction factor (VRF) - namely, the sample variance of $\hat{\mu}_{n_{\text {gen }}}^{\mathrm{NN}, \mathrm{MC}}$ over the sample variance of $\hat{\mu}_{n_{\text {gen }}}^{\mathrm{NN}}$ - and the corresponding improvement in percentages.

From Figure 13 , we observe that $\hat{\mu}_{n_{\text {gen }}}^{\mathrm{NN}}$ is able to reduce the variance in all considered applications and across all dimensions. While variance reduction is diminished in higher dimensions $(d=10)$, the GMMN RQMC estimator is still immensely useful in estimating expectations $\mu$ for three reasons. Firstly, as demonstrated in the previous section, GMMNs best fit the underlying dependence structure of the data. Secondly, unlike many parametric copulas, we can generate quasi-random samples independent of the type of dependence structure observed in the data. Finally, we can generate GMMN quasi-random samples at no additional cost over GMMN pseudo-random samples; see also Appendix B. 

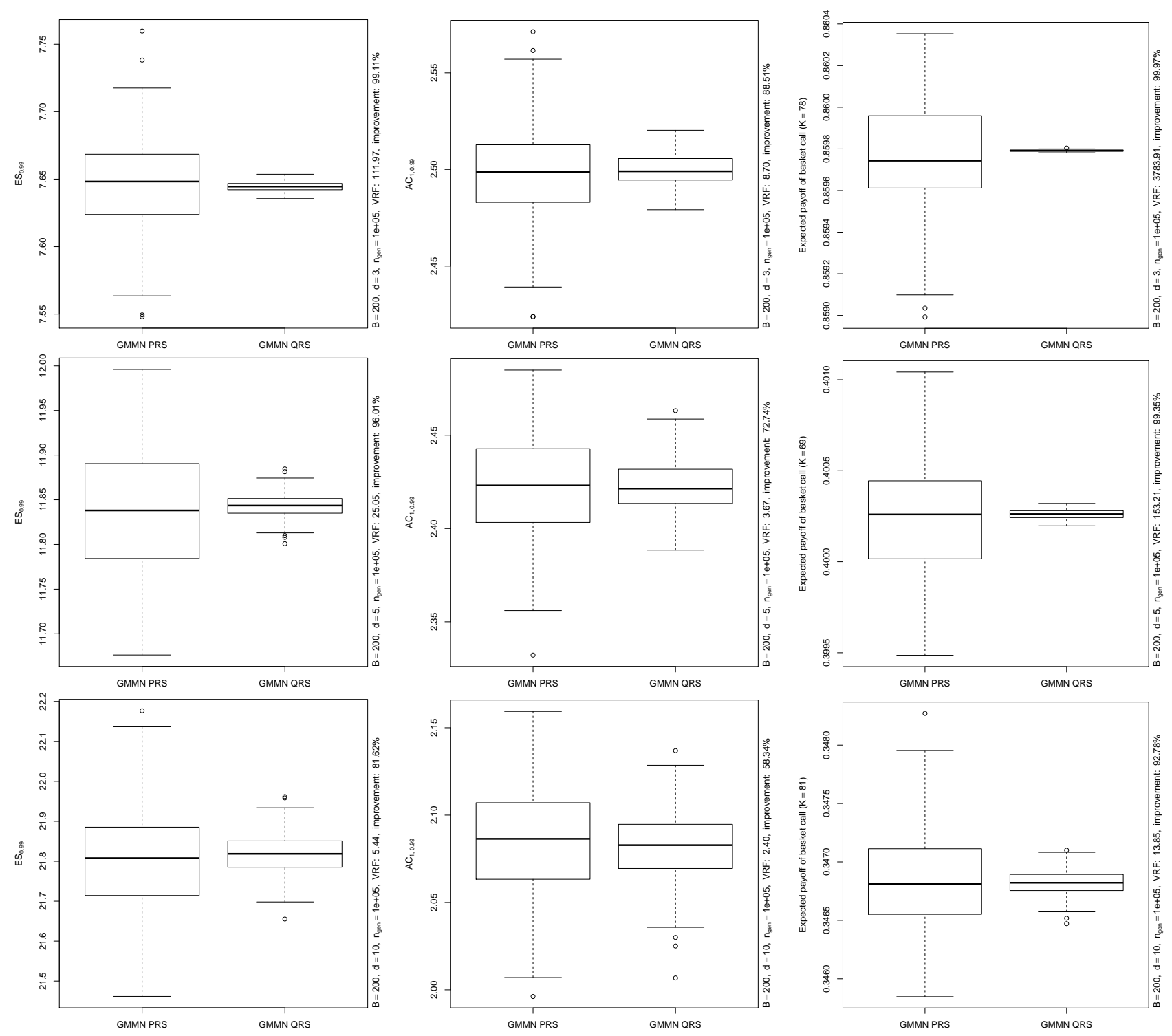

Figure 13 Box plots based on $B=200$ realizations of the GMMN MC estimator $\hat{\mu}_{n_{\text {gen }}}^{\mathrm{NN}, \mathrm{MC}}$ (label "GMMN PRS") and the GMMN RQMC estimator $\hat{\mu}_{n_{\text {gen }}}^{\mathrm{NN}}$ (label "GMMN QRS") of $\mathrm{ES}_{0.99}$ (left column), $\mathrm{AC}_{1,0.99}$ (middle column) and the expected payoff of a basket call (right column) for portfolio with dimensions $d=3$ (top row), $d=5$ (middle row) and $d=10$ (bottom), using $n_{\text {gen }}=10^{5}$ samples for both estimators. 


\section{Discussion}

\section{Discussion}

This work has been inspired by the simple question of how to obtain quasi-random samples for a large variety of multivariate distributions. Until recently, this was only possible for a few multivariate distributions with specific underlying copulas. In general, for the vast majority of multivariate distributions, obtaining quasi-random samples is a hard problem (Cambou et al., 2017). Our approach based on GMMNs provides a first universal method for doing so. It depends on first learning a generator $f_{\hat{\boldsymbol{\theta}}}$ such that, given $\boldsymbol{Z}$ (with independent components from some known distribution such as the standard uniform or standard normal), $f_{\hat{\boldsymbol{\theta}}}(\boldsymbol{Z})$ follows the targeted multivariate distribution. Conditional on this first step being successful, we can then replace $\boldsymbol{Z}$ with $F_{\boldsymbol{Z}}^{-1}\left(\tilde{\boldsymbol{v}}_{i}\right), i=1, \ldots, n_{\text {gen }}$, where $\left\{\tilde{\boldsymbol{v}}_{1}, \ldots, \tilde{\boldsymbol{v}}_{n_{\text {gen }}}\right\}$ is an RQMC point set, to generate quasi-random samples from $\boldsymbol{X}$.

It is generally difficult to assess the low-discrepancy property of non-uniform quasi-random samples. To evaluate the quality of our GMMN quasi-random samples, we used visualization tools (Section 3.2), goodness-of-fit statistics (Section 3.3), and investigated variance reduction effects (Section 4) when estimating $\mu=\mathbb{E}(\Psi(\boldsymbol{X}))$ for a test function and for expected shortfall. As dependence structures among the components of $\boldsymbol{X}$, we included various known copulas, some of which allowed for quasirandom sampling which allowed us to statistically assess the performance of our GMMN quasi-random samples. However, we emphasize that the key feature of our method is that, given a sufficiently large dataset with dependence structure not well described by any known parametric copula model for which quasi-random sampling is available, we are now able to generate quasi-random samples from its empirical distribution. We demonstrated this with a real dataset in Section 5 . Not only does a GMMN provide the best fitting model in this application, allowing us to avoid the tedious and often computationally challenging search that is typically required in classical copula modeling for an adequate dependence model, we also obtain, at no additional cost, quasi-random samples from this GMMN - a whole other challenge in classical copula modeling. This universality and computability is an attractive feature of GMMNs for multivariate modeling.

However, this does not mean that the problem of quasi-random sampling for multivariate distributions is completely solved. In high dimensions learning an entire distribution is a hard problem, and so is learning the generator $f_{\hat{\boldsymbol{\theta}}}$. At a superficial level, the literature on generative NNs - and the many headlines covering them - may give the impression that such NNs are now capable of generating samples from very high-dimensional distributions. This, of course, is not true; see, for example, Arjovsky et al. (2017), S. Arora et al. (2018), and Tolstikhin et al. (2017). In particular, while available evidence is convincing that any specific generated sample $f_{\hat{\boldsymbol{\theta}}}\left(\boldsymbol{Z}_{1}\right)$, typically an image, can be very realistic in the sense that it looks just like a typical training sample, this is not the same as saying that the entire collection of generated samples $\left\{f_{\hat{\boldsymbol{\theta}}}\left(\boldsymbol{Z}_{1}\right), f_{\hat{\boldsymbol{\theta}}}\left(\boldsymbol{Z}_{2}\right), \ldots\right\}$ will have the same distribution as the training sample. The latter is a much harder problem to solve. Unlike widely cited generative NNs such as variational autoencoders and generative adversarial networks, GMMNs are capable of learning entire distributions, because they rely on the MMD-criterion as the cost function rather than, for example, the mean squared error which does not measure the discrepancy between entire distributions. Even so, this still does not mean GMMNs are practical for very high dimensions yet, simply because the fundamental curse of dimensionality cannot be avoided easily. At the moment, it is simply not realistic to hope that one can learn an entire distribution in high dimensions from a training sample of only moderate size.

Going forward there are two primary impediments to quasi-random sampling from higher-dimensional copulas and distributions. Firstly, the problem of distribution learning via generative NNs remains a challenging task. We may also consider using other goodness-of-fit statistics for multivariate distributions rather than the MMD as the cost function (provided that the statistic is differentiable in 
order to train a generative NN). Secondly, we discovered from our empirical investigation in Section 4 that the convergence rates of GMMN RQMC estimators decrease with increasing dimension. Preserving the low-discrepancy of RQMC point sets upon transformations in high dimensions remains an open problem in this regard.

\section{A Analyzing GMMN QMC and GMMN RQMC estimators}

\section{A.1 QMC point sets}

The idea behind quasi-random numbers is to replace pseudo- $U(0,1)^{p}$ random numbers with lowdiscrepancy points sets $P_{n_{\text {gen }}}$ to produce a more homogeneous coverage of $[0,1]^{p}$ in comparison to pseudo-random numbers. That is, with respect to a certain discrepancy measure, the empirical distribution of the $P_{n_{\text {gen }}}$ is closer to the uniform distribution $\mathrm{U}(0,1)^{p}$ than a pseudo-random sample.

Established notions of the discrepancy of a point set $P_{n_{\text {gen }}}$ are as follows. The discrepancy function of $P_{n_{\text {gen }}}$ in an interval $I=[\mathbf{0}, \boldsymbol{b})=\prod_{j=1}^{p}\left[0, b_{j}\right), b_{j} \in(0,1], j=1, \ldots, p$, is defined by

$$
D\left(I ; P_{n_{\text {gen }}}\right)=\frac{1}{n_{\text {gen }}} \sum_{i=1}^{n_{\text {gen }}} \mathbb{1}_{\left\{\boldsymbol{v}_{i} \in I\right\}}-\lambda(I),
$$

where $\lambda(I)$ is the $p$-dimensional Lebesgue measure of $I$. Thus the discrepancy function is the difference between the number of points of $P_{n_{\text {gen }}}$ in $I$ and the probability of a $p$-dimensional standard uniform random vector to fall in $I$. For $\mathcal{A}=\left\{[\mathbf{0}, \boldsymbol{b}): \boldsymbol{b} \in(0,1]^{p}\right\}$, the star discrepancy of $P_{n_{\text {gen }}}$ is defined by

$$
D^{*}\left(P_{n_{\text {gen }}}\right)=\sup _{I \in \mathcal{A}}\left|D\left(I ; P_{n_{\text {gen }}}\right)\right| .
$$

If $P_{n_{\text {gen }}}$ satisfies the condition $D^{*}\left(P_{n_{\text {gen }}}\right) \in O\left(n_{\text {gen }}^{-1}\left(\log n_{\text {gen }}\right)^{p}\right)$, it is called a low-discrepancy sequence (Lemieux, 2009, p. 143).

There are different approaches to construct (deterministic) low-discrepancy sequences; see Lemieux (2009, Chapters 5-6). The two main approaches involve either lattices (grids which behave well under projections) or digital nets/sequences. In our numerical investigations presented in Sections 3 4, we worked with a type of digital net constructed using the Sobol' sequence; see Sobol' (1967).

\section{A.2 Analyzing the GMMN QMC estimator}

In this section, we will derive conditions under which the (non-randomized) GMMN QMC estimator

$$
\frac{1}{n_{\text {gen }}} \sum_{i=1}^{n_{\text {gen }}} \Psi\left(q\left(\boldsymbol{v}_{i}\right)\right)=\frac{1}{n_{\text {gen }}} \sum_{i=1}^{n_{\text {gen }}} h\left(\boldsymbol{v}_{i}\right)
$$

where $q=f_{\hat{\boldsymbol{\theta}}} \circ F_{\boldsymbol{Z}}^{-1}$ and $h=\Psi \circ q=\Psi \circ f_{\hat{\boldsymbol{\theta}}} \circ F_{\boldsymbol{Z}}^{-1}$, has a small error when approximating $\mathbb{E}(\Psi(\boldsymbol{Y}))$. In the following analysis, we need to further assume that $\operatorname{supp}\left(F_{\boldsymbol{X}}\right)$ and $\operatorname{supp}\left(F_{\boldsymbol{Y}}\right)$ are bounded.

The Koksma-Hlawka inequality (Niederreiter, 1992) for a function $g:[0,1]^{p} \rightarrow \mathbb{R}$ says that

$$
\left|\frac{1}{n_{\text {gen }}} \sum_{i=1}^{n_{\text {gen }}} g\left(\boldsymbol{v}_{i}\right)-\mathbb{E}\left(g\left(\boldsymbol{U}^{\prime}\right)\right)\right| \leq V(g) D^{*}\left(P_{n_{\text {gen }}}\right),
$$

where $\boldsymbol{U}^{\prime} \sim \mathrm{U}(0,1)^{p}$ and the variation $V(g)$ is understood in the sense of Hardy and Krause; we refer to the right-hand side of the inequality as Koksma-Hlawka bound. From this Koksma-Hlawka inequality, 
we can establish that if $g$ has finite bounded variation, that is $V(g)<\infty$, then the convergence rate for $\frac{1}{n_{\text {gen }}} \sum_{i=1}^{n_{\text {gen }}} g\left(\boldsymbol{v}_{i}\right)$ is determined by $D^{*}\left(P_{n_{\text {gen }}}\right)=O\left(n_{n_{\text {gen }}}^{-1}\left(\log n_{\text {gen }}\right)^{p}\right)$.

We can use the Koksma-Hlawka inequality to analyze the convergence of the GMMN QMC estimator $\frac{1}{n_{\text {gen }}} \sum_{i=1}^{n_{\text {gen }}} \Psi\left(\boldsymbol{y}_{i}\right)$ of $\mathbb{E}(\Psi(\boldsymbol{Y}))$, where $\boldsymbol{y}_{i}=q\left(\boldsymbol{v}_{i}\right), i=1, \ldots, n_{\text {gen }}$ and $\boldsymbol{Y} \sim F_{\boldsymbol{Y}}$, by establishing the conditions under which $V(h)$ is bounded. To that end, consider the following proposition.

\section{Proposition A.1 (Sufficient conditions for finiteness of the Koksma-Hlawka bound)}

Assume that $\operatorname{supp}\left(F_{\boldsymbol{Y}}\right)$ is bounded and all appearing partial derivatives of $q$ and $\Psi$ exist and are continuous. Consider $q=f_{\hat{\boldsymbol{\theta}}} \circ F_{\boldsymbol{Z}}^{-1}$, the point set $P_{n_{\text {gen }}}=\left\{\boldsymbol{v}_{1}, \ldots, \boldsymbol{v}_{n_{\text {gen }}}\right\} \subseteq[0,1)^{p}$ and let $\boldsymbol{y}_{i}=q\left(\boldsymbol{v}_{i}\right)$, $i=1, \ldots, n_{\text {gen }}$, denote the GMMN quasi-random sample. Suppose that

1) $\Psi(\boldsymbol{y})<\infty$ for all $\boldsymbol{y} \in \operatorname{supp}\left(F_{\boldsymbol{Y}}\right)$ and

$$
\frac{\partial^{|\boldsymbol{\beta}|_{1}} \Psi(\boldsymbol{y})}{\partial^{\beta_{1}} y_{1} \ldots \partial^{\beta_{d}} y_{d}}<\infty, \quad \boldsymbol{y} \in \operatorname{supp}\left(F_{\boldsymbol{Y}}\right)
$$

for all $\boldsymbol{\beta}=\left(\beta_{1}, \ldots, \beta_{d}\right) \subseteq\{0, \ldots, d\}^{d}$ and $|\boldsymbol{\beta}|_{1}=\sum_{j=1}^{d} \beta_{j} \leq d ;$

2) there exists an $M>0$ such that $\left|\mathrm{D}^{k} F_{Z_{j}}^{-1}\right| \leq M$, for each $k, j=1, \ldots, p$, where $\mathrm{D}^{k}$ denotes the $k$-fold derivative of its argument;

3) there exists, for each layer $l=1, \ldots, L+1$ of the $\mathrm{NN} f_{\hat{\boldsymbol{\theta}}}$, an $N_{l}>0$ such that $\left|\mathrm{D}^{k} \phi_{l}\right| \leq N_{l}$ for all $k=1, \ldots, p ;$ and

4) the parameter vector $\hat{\boldsymbol{\theta}}=\left(\widehat{W}_{1}, \ldots, \widehat{W}_{L+1}, \hat{\boldsymbol{b}}_{1}, \ldots, \hat{\boldsymbol{b}}_{L+1}\right)$ of the fitted NN is bounded.

Then there exists a constant $c$ independent of $n_{\text {gen }}$, but possibly depending on $\Psi, \hat{\boldsymbol{\theta}}, M$ and $N_{1}, \ldots, N_{L+1}$, such that

$$
\left|\frac{1}{n_{\text {gen }}} \sum_{i=1}^{n_{\text {gen }}} \Psi\left(\boldsymbol{y}_{i}\right)-\mathbb{E}(\Psi(\boldsymbol{Y}))\right| \leq c D^{*}\left(P_{n_{\text {gen }}}\right) .
$$

Proof. To begin with note that for any $q$ such that $q\left(\boldsymbol{U}^{\prime}\right) \sim F_{\boldsymbol{Y}}$, we know that $\boldsymbol{Y}$ is in distribution equal to $q\left(\boldsymbol{U}^{\prime}\right)$ and thus $\mathbb{E}(\Psi(\boldsymbol{Y}))=\mathbb{E}\left(\Psi\left(q\left(\boldsymbol{U}^{\prime}\right)\right)\right)=\mathbb{E}\left(h\left(\boldsymbol{U}^{\prime}\right)\right)$. Based on this property, we can obtain the Koksma-Hlawka bound $V(h) D^{*}\left(P_{n_{\text {gen }}}\right)$ for the change of variable $h$.

Following Lemieux (2009, Section 5.6.1), we can derive an expression for $V(h)$. To this end, let

$$
V^{(j)}(h ; \boldsymbol{\alpha})=\int_{[0,1)^{j}}\left|\frac{\partial^{j} h^{(\boldsymbol{\alpha})}\left(v_{\alpha_{1}}, \ldots, v_{\alpha_{j}}\right)}{\partial v_{\alpha_{j}} \ldots \partial v_{\alpha_{1}}}\right| \mathrm{d} v_{\alpha_{1}} \ldots \mathrm{d} v_{\alpha_{j}}
$$

where $h^{(\boldsymbol{\alpha})}\left(v_{\alpha_{1}}, \ldots, v_{\alpha_{j}}\right)=h\left(\tilde{v}_{1}, \ldots, \tilde{v}_{p}\right)$ for $\tilde{v}_{k}=v_{k}$ if $k \in\left\{\alpha_{1}, \ldots, \alpha_{j}\right\}$ and $\tilde{v}_{k}=1$ otherwise. Then

$$
V(h)=\sum_{j=1}^{p} \sum_{\boldsymbol{\alpha}:|\boldsymbol{\alpha}|_{1}=j} V^{(j)}(h ; \boldsymbol{\alpha}),
$$

where the inner sum is taken over all $\boldsymbol{\alpha}=\left(\alpha_{1}, \ldots, \alpha_{j}\right)$ with $\left\{\alpha_{1}, \ldots, \alpha_{j}\right\} \subseteq\{1, \ldots, p\}-$ see also Niederreiter (1992, pp. 19-20), E. Hlawka (1961, eq. (4)) and Hlawka and Mück (1972, eq. (4')). Following Hlawka and Mück (1972) and Constantine and Savits (1996, Theorem 2.1), we then have

$$
\left|\frac{\partial^{j} h^{(\boldsymbol{\alpha})}\left(v_{\alpha_{1}}, \ldots, v_{\alpha_{j}}\right)}{\partial v_{\alpha_{j}} \ldots \partial v_{\alpha_{1}}}\right|=\sum_{1 \leq|\boldsymbol{\beta}|_{1} \leq j} \frac{\partial^{|\boldsymbol{\beta}|_{1}} \Psi}{\partial^{\beta_{1}} y_{1} \ldots \partial^{\beta_{d}} y_{d}} \sum_{i=1}^{j} \sum_{(\boldsymbol{\kappa}, \boldsymbol{k}) \in \pi_{i}(\boldsymbol{\kappa}, \boldsymbol{k})} c_{\boldsymbol{\kappa}} \prod_{m=1}^{i} \frac{\partial^{\left|\boldsymbol{\kappa}_{m}\right|_{1}} q_{k_{m}}^{(\boldsymbol{\alpha})}\left(v_{\alpha_{1}}, \ldots, v_{\alpha_{j}}\right)}{\partial^{\kappa_{m j}} v_{\alpha_{j}} \ldots \partial^{\kappa_{m 1}} v_{\alpha_{1}}}
$$


where $\boldsymbol{\beta} \in \mathbb{N}_{0}^{d}$ and where $\pi_{i}(\boldsymbol{\kappa}, \boldsymbol{k})$ denotes the set of pairs $(\boldsymbol{\kappa}, \boldsymbol{k})$ such that $\boldsymbol{k}=\left(k_{1}, \ldots, k_{i}\right) \in\{1, \ldots, d\}^{i}$ and $\boldsymbol{\kappa}=\left(\boldsymbol{\kappa}_{1}, \ldots, \boldsymbol{\kappa}_{i}\right)$ with $\boldsymbol{\kappa}_{m}=\left(\kappa_{m 1}, \ldots, \kappa_{m j}\right) \in\{0,1\}^{j}, m=1, \ldots, i$, and $\sum_{m=1}^{i} \kappa_{m i}=1$ for $i=1, \ldots, j$; see Constantine and Savits $(1996)$ for more details on $\pi_{i}(\boldsymbol{\kappa}, \boldsymbol{k})$ and the constants $c_{\kappa}$. Furthermore, for index $j=1, \ldots, d, q_{j}^{(\alpha)}\left(v_{\alpha_{1}}, \ldots, v_{\alpha_{j}}\right)=q_{j}\left(\tilde{v}_{1}, \ldots, \tilde{v}_{p}\right)$ and $q_{j}\left(\tilde{v}_{1}, \ldots, \tilde{v}_{p}\right)=$ $\phi_{L+1}\left(\widehat{W}_{L+1 j} . \boldsymbol{a}_{L}+\hat{\boldsymbol{b}}_{L+1}\right)$, where $\boldsymbol{a}_{l}=\phi_{l}\left(\widehat{W}_{l} \boldsymbol{a}_{l-1}+\hat{\boldsymbol{b}}_{l}\right)$ for $l=1, \ldots, L$ with $\boldsymbol{a}_{0}=F_{\boldsymbol{Z}}^{-1}(\tilde{\boldsymbol{v}})$ and where $\widehat{W}_{L+1 j}$. denotes the $j$ th row of $W_{L+1}$.

Based on the decomposition in (13), a sufficient condition to ensure that $V(h)<\infty$ is that all products of the form

$$
\frac{\partial^{|\boldsymbol{\beta}|_{1}} \Psi}{\partial^{\beta_{1}} y_{1} \ldots \partial^{\beta_{d}} y_{d}} \prod_{m=1}^{i} \frac{\partial^{\left|\kappa_{m}\right|_{1}} q_{k_{m}}^{(\boldsymbol{\alpha})}\left(v_{\alpha_{1}}, \ldots, v_{\alpha_{j}}\right)}{\partial^{\kappa_{m j}} v_{\alpha_{j}} \ldots \partial^{\kappa_{m 1}} v_{\alpha_{1}}}, \quad i=1, \ldots, j
$$

are integrable.

To that end, Assumptions 2) 4) imply that all mixed partial derivatives of $q=f_{\hat{\boldsymbol{\theta}}} \circ F_{\boldsymbol{Z}}^{-1}$ are bounded. By the assumption of continuous partial derivatives of $q$, this implies that finite products of the form

$$
\prod_{m=1}^{i} \frac{\partial^{\left|\kappa_{m}\right|_{1}} q_{k_{m}}^{(\boldsymbol{\alpha})}\left(v_{\alpha_{1}}, \ldots, v_{\alpha_{j}}\right)}{\partial^{\kappa_{m j}} v_{\alpha_{j}} \ldots \partial^{\kappa_{m 1}} v_{\alpha_{1}}}, \quad i=1, \ldots, j
$$

are integrable. By Assumption 1), decomposition (13) and Hölder's inequality, the quantity in (12) is bounded. This implies that $h$ has bounded variation, so that the Koksma-Hlawka bound is finite.

The following remark provides insights into Assumptions 2) 4 ) of Proposition A.1

\section{Remark A.2}

$\mathrm{U}(a, b)^{p}$ for $a<b$, which is a popular choice for the input distribution, clearly satisfies Assumption 2) in Proposition A.1. Assumption 3) is satisfied for various commonly used activation functions, such as:

1) Sigmoid. If $\phi_{l}(x)=1 /\left(1+\mathrm{e}^{-x}\right)$ for layer $l$, then $N_{l}=1$.

2) ReLU. If $\phi_{l}(x)=\max \{0, x\}$ for layer $l$, then $N_{l}=1$. In this case, only the first derivative is (partly) non-zero. Additionally, note that the ReLU activation function is not differentiable at $x=0$. However, even if $\phi_{l}=\max \{0, x\}$ for all $l=1, \ldots, L+1$, the set of all pointwise discontinuities of the mixed partial derivatives of $q$ is a null set. Hence, the discontinuities do not jeopardize the proof of Proposition A.1.

3) Softplus. If $\phi_{l}(x)=\log \left(1+\mathrm{e}^{x}\right)$ for layer $l$, then $N_{l}=1$. The Softplus activation function can be used as a smooth approximation of the ReLU activation function.

4) Linear. If $\phi_{l}(x)=x$ for layer $l$, then $N_{l}=1$. Only the first derivative is non-zero.

5) Tanh. If $\phi_{l}(x)=\tanh (x)$ for layer $l$, then $N_{l}=1$.

6) Scaled exponential linear unit (SELU); see Klambauer et al. (2017). If, for layer $l$,

$$
\phi_{l}(x)= \begin{cases}\lambda \alpha(\exp (-x)-1), & \text { if } x<0 \\ \lambda x, & \text { if } x \geq 0\end{cases}
$$

where $\lambda$ and $\alpha$ are prespecified constants, then $N_{l}=\max \{\lambda, \lambda \alpha, 1\}$. The same argument about discontinuities made with the ReLU activation function applies equally well to the case of the SELU activation function. 


\section{A Analyzing GMMN QMC and GMMN RQMC estimators}

Assumption 4) of Proposition A.1 is satisfied in practice because NNs are always trained with regularization on the parameters, which means $\hat{\boldsymbol{\theta}}$ always lies in a compact set. Additionally note that in the general case where $q$ is characterized by a composition of NN layers and $F_{Z}^{-1}$ with a different (but standard) activation function in each layer, all partial derivatives of $q$ exist and are continuous. Moreover, for the activation functions and input distributions listed above, all mixed partial derivatives of $q$ are bounded.

\section{A.3 RQMC point sets}

In Monte Carlo applications, we need to randomize the low-discrepancy sequence $P_{n_{\text {gen }}}$ to obtain unbiased estimators and variance estimates. To that end, we can randomize $P_{n_{\text {gen }}}$ via a $U^{\prime} \sim \mathrm{U}(0,1)^{p}$ to obtain a randomized point set $\tilde{P}_{n_{\text {gen }}}=\tilde{P}_{n_{\text {gen }}}\left(\boldsymbol{U}^{\prime}\right)=\left\{\tilde{\boldsymbol{v}}_{1}, \ldots, \tilde{\boldsymbol{v}}_{n_{\text {gen }}}\right\}$, where $\tilde{\boldsymbol{v}}_{i}=r\left(\boldsymbol{U}^{\prime}, \boldsymbol{v}_{i}\right), i=1, \ldots, n_{\text {gen }}$, for a certain randomization function $r$. A simple randomization to obtain an RQMC point set is to consider $\tilde{\boldsymbol{v}}_{i}=\left(\boldsymbol{v}_{i}+\boldsymbol{U}^{\prime}\right) \bmod 1, i=1, \ldots, n_{\text {gen }}$, for $\boldsymbol{U}^{\prime} \sim \mathrm{U}(0,1)^{p}$, a so-called random shift; see Cranley and Patterson (1976).

In practice, more sophisticated alternatives to the random shift are often used. One such slightly more sophisticated randomization scheme is the digital shift method; see Lemieux (2009, Chapter 6) and Cambou et al. (2017). In the same vein as the random shift, one adds a random uniform shift to points in $P_{n_{\text {gen }}}$, but with operations in $\mathbb{Z}_{b}$, where $b$ is the base in which the digital net is defined, rather than simply adding two real numbers. We use $\tilde{P}_{n_{\text {gen }}}^{\text {ds }}$ to denote the RQMC point set obtained using the digital shift method.

Another randomization approach is to scramble the digital net. This technique was originally proposed by Owen (1995). In particular, the type of scrambling we work with is referred to as the nested uniform scrambling (or full random scrambling) method; see Owen (2003). Since we primarily use this method throughout the paper, $\tilde{P}_{n_{\text {gen }}}$ will denote specifically the RQMC point set obtained using scrambling. The digital shift method is more computationally efficient in comparison to scrambling but because the distortion of the deterministic point set is fairly simple in the digital shift method, there are bad functions one can construct such that the variance of the RQMC estimator is larger than that of the corresponding MC estimator; see Lemieux (2009, Chapter 6). Furthermore, when RQMC points are constructed with scrambling, we can justify (see Appendix A.4 that an improved rate of $O\left(n_{\text {gen }}^{-3}\left(\log n_{\text {gen }}\right)^{p-1}\right)$ is achievable for $\operatorname{Var}\left(\hat{\mu}_{n_{\text {gen }}}^{\mathrm{NN}}\right)$; this translates to $O\left(n_{\text {gen }}^{-3 / 2}\left(\log n_{\text {gen }}\right)^{(p-1) / 2}\right)$ on the root mean squared error (RMSE) scale, which is more directly comparable to the convergence rate of $O\left(n_{\text {gen }}^{-1}\left(\log n_{\text {gen }}\right)^{p}\right)$ implied by the Koksma-Hlawka bound for the mean absolute error (MAE) of $\hat{\mu}_{n_{\text {gen }}}^{\mathrm{NN}}$ using QMC points (see Appendix A.2). Hence, even though the aforementioned bad functions do not often arise in practice, we primarily work with the scrambling randomization method to construct our RQMC point sets. Both the scrambling and the digital shift methods are available in the $\mathrm{R}$ package qrng and can be accessed via $\operatorname{sobol}($, randomize $=$ "Owen") and $\operatorname{sobol}($, randomize = "digital.shift") respectively.

The randomization schemes discussed above preserve the low-discrepancy property of $P_{n_{\text {gen }}}$ and the estimators of interest obtained using each type of RQMC point set are unbiased. Computing the estimator based on $B$ such randomized point sets and computing the sample variance of the resulting $B$ estimates then allows us to estimate the variance of the estimator of interest. 


\section{A.4 Analyzing the GMMN RQMC estimator}

\section{A.4.1 GMMN RQMC estimators constructed with scrambled nets}

For RQMC estimators $\frac{1}{n_{\text {gen }}} \sum_{i=1}^{n_{\text {gen }}} g(\tilde{\boldsymbol{v}})$ based on scrambled nets, Owen (1997) initially derived a convergence rate for the variance of the estimators under a certain smoothness condition on $g$, where $g:[0,1]^{p} \rightarrow \mathbb{R}$. Owen $(2008)$ then generalized his earlier result to allow a weaker smoothness condition for a larger class of scrambled nets. Specifically, if $\tilde{P}_{n_{\text {gen }}}=\left\{\tilde{\boldsymbol{v}}_{1}, \ldots, \tilde{\boldsymbol{v}}_{n_{\text {gen }}}\right\}$ is a so-called relaxed scrambled $(\lambda, q, m, p)$-net in base $b$ with bounded gain coefficients - for example, Sobol' sequences randomized using nested uniform sampling belong to this class - then we have the following result as a direct consequence of Owen (2008).

Theorem A.3 (Owen (2008))

If all the mixed partial derivatives (up to order $p$ ) of $g$ exist and are continuous, then

$$
\operatorname{Var}\left(\frac{1}{n_{\text {gen }}} \sum_{i=1}^{n_{\text {gen }}} g\left(\tilde{\boldsymbol{v}}_{i}\right)\right)=O\left(n_{\text {gen }}^{-3}\left(\log n_{\text {gen }}\right)^{p-1}\right) .
$$

Proof. See Owen (2008, Theorem 3).

Now, for the GMMN RQMC estimator, $\hat{\mu}_{n_{\text {gen }}}^{\mathrm{NN}}=\frac{1}{n_{\text {gen }}} \sum_{i=1}^{n_{\text {gen }}} \Psi\left(q\left(\tilde{\boldsymbol{v}}_{i}\right)\right)=\frac{1}{n_{\text {gen }}} \sum_{i=1}^{n_{\text {gen }}} h\left(\tilde{\boldsymbol{v}}_{i}\right)$, the corollary below naturally follows from Theorem A.3 with some added analysis of the composite function $h$.

\section{Corollary A.4}

If all the mixed partial derivatives (up to order $p$ ) of $h=\Psi \circ q=\Psi \circ f_{\hat{\boldsymbol{\theta}}} \circ F_{\boldsymbol{Z}}^{-1}$ exist and are continuous, then $\operatorname{Var}\left(\hat{\mu}_{n_{\text {gen }}}^{\mathrm{NN}}\right)=O\left(n_{\text {gen }}^{-3}\left(\log n_{\text {gen }}\right)^{p-1}\right)$.

To analyze the mixed partial derivatives of $h$, it suffices to analyze each component function separately.

For popular choices of input distributions (such as $\mathrm{U}(a, b)^{p}$ for $a<b$ or $\left.\mathrm{N}(0,1)^{p}\right)$, the $k$-fold derivative $\mathrm{D}^{k} F_{Z_{j}}^{-1}$ exists and is continuous (on $[a, b]$ or $\mathbb{R}$ depending on the choice of input distribution) for each $k, j=1, \ldots, p$.

For each layer $l=1, \ldots, L+1$ of the $\mathrm{NN} f_{\hat{\boldsymbol{\theta}}}, \mathrm{D}^{k} \phi_{l}$ exists and is continuous for $k=1, \ldots, p$ provided that we use (standard) activation functions; see Remark A.2 for further details on suitable activation functions. For NNs constructed using some popular activation functions such as the ReLU and SELU, note that the set of all pointwise discontinuities of the mixed partial derivatives of $f_{\hat{\boldsymbol{\theta}}}$ is a set of Lebesgue measure zero and hence the proof of Theorem A.3 holds. Alternatively, we can use the softplus activation function as a smoother approximation of ReLU. Now in the most general case of NNs $f_{\hat{\boldsymbol{\theta}}}$ being composed of layers with different (but standard) activation functions, all mixed partial derivatives (up to order $p$ ) of $f_{\hat{\boldsymbol{\theta}}}$ exist and are continuous almost everywhere.

Finally, it is certainly true that, for many functionals $\Psi$ that we care about in practice, such as those considered in Sections 4 and 5.3, all of its mixed partial derivatives (up to order $p$ ) exist and are continuous almost everywhere on $\mathbb{R}^{d}$.

\section{A.4.2 GMMN RQMC estimators constructed with digitally shifted nets}

For GMMN RQMC estimators $\hat{\mu}_{n_{\text {gen }}}^{\mathrm{NN} \text {,ds }}$ constructed using digitally shifted RQMC point sets $\tilde{P}_{n_{\text {gen }}}^{\text {ds }}$, we can obtain an expression for $\operatorname{Var}\left(\hat{\mu}_{n_{\text {gen }}}^{\mathrm{NN}, \mathrm{ds}}\right)$ under the condition that the composite function $h$ is square integrable; see Cambou et al. (2017, Proposition 6).

With added assumptions on the smoothness of $h$, one can obtain improved convergence rates (compared to MC estimators) for $\operatorname{Var}\left(\hat{\mu}_{n_{\text {gen }}}^{\mathrm{NN}, \mathrm{ds}}\right)$. For example, under the assumptions of Proposition A.1. 


\section{B Run time}

$h$ has finite bounded variation in the sense of Hardy-Krause, which implies that $\operatorname{Var}\left(\hat{\mu}_{n_{\text {gen }}}^{\mathrm{NN}, \mathrm{ds}}\right)=$ $O\left(n_{\text {gen }}^{-2}\left(\log n_{\text {gen }}\right)^{2 p}\right)$; see L'Ecuyer $(2016)$.

In practice, we observe that GMMN RQMC estimators constructed using both scrambled and digitally shifted nets achieve very similar convergence rates despite differences in the theoretical convergence rates. To that end, Figure 14 shows plots of standard deviation estimates for estimating $\mathbb{E}\left(\Psi_{2}(\boldsymbol{X})\right)$ where we use the RQMC point sets $\tilde{P}_{n_{\text {gen }}}^{\text {ds }}$ for the same copula models as considered for Figure 11 (which is based on GMMN RQMC estimators constructed using scramble nets) in Section 4 The approximate convergence rates as implied by the regression coefficients $\alpha$ displayed in both figures are very similar across the various examples.

\section{B Run time}

Run time depends on factors such as the hardware used, the current workload, the algorithm implemented, the programming language used, the implementation style, compiler flags, whether garbage collection was used, etc. There is not even a unique concept of time (system vs user vs elapsed time). Although none of our code was optimized for run time, we still report on various timings here, measured in elapsed time also known as wall-clock time.

\section{B.1 Training and sampling}

The results in this section are reproducible with the demo GMMN_QMC_timings of the R package gnn.

Table 1 shows elapsed times in minutes for training a GMMN on training data from $t_{4}$, Clayton $(\mathrm{C})$, Gumbel (G) and nested Gumbel (NG) copulas in dimensions 2, 3, 5 and 10 as described in Sections 3.2 and 3.3. As is reasonable, the measured times are only affected by the dimension, not by the type of dependence model.

\begin{tabular}{cccc}
\hline$C$ & $d=2,3$ & $d=5$ & $d=10$ \\
\hline$t_{4}$ & 5.52 & 7.01 & 9.46 \\
$\mathrm{C}$ & 5.52 & 7.00 & 9.45 \\
$\mathrm{G}$ & 5.52 & 7.01 & 9.46 \\
$\mathrm{NG}$ & 6.01 & 7.01 & 9.44 \\
\hline
\end{tabular}

Table 1 Elapsed times in minutes for training GMMNs of the same architecture as used in Sections 3.2 and 3.3 with $n_{\text {epo }}=300, n_{\text {trn }}=60000$ and $n_{\text {bat }}=5000$ on respective copula samples; training was done on one NVIDIA Tesla P100 GPU.

Table 2 contains elapsed times for generating $n_{\text {gen }}=10^{5}$ observations from the respective dependence model and sampling method on two different machines, once on the NVIDIA Tesla P100 GPU used for training and once locally on a $20182.7 \mathrm{GHz}$ Quad-Core Intel Core i7 processor. The results for the copula-based pseudo-random sampling method are averaged over 100 repetitions. The results for the copula-based quasi-random sampling method are obtained as follows. If the conditional copulas involved in applying the inverse Rosenblatt transform of the respective copula model are not available analytically nor numerically, NA is reported; this applies to the nested Gumbel copula. And if they are only available numerically (by root finding), then a reduced sample size of 1000 is used and the reported run times were obtained by scaling up to $n_{\text {gen }}$ by multiplication with 100; this applies to the Gumbel 


\section{B Run time}
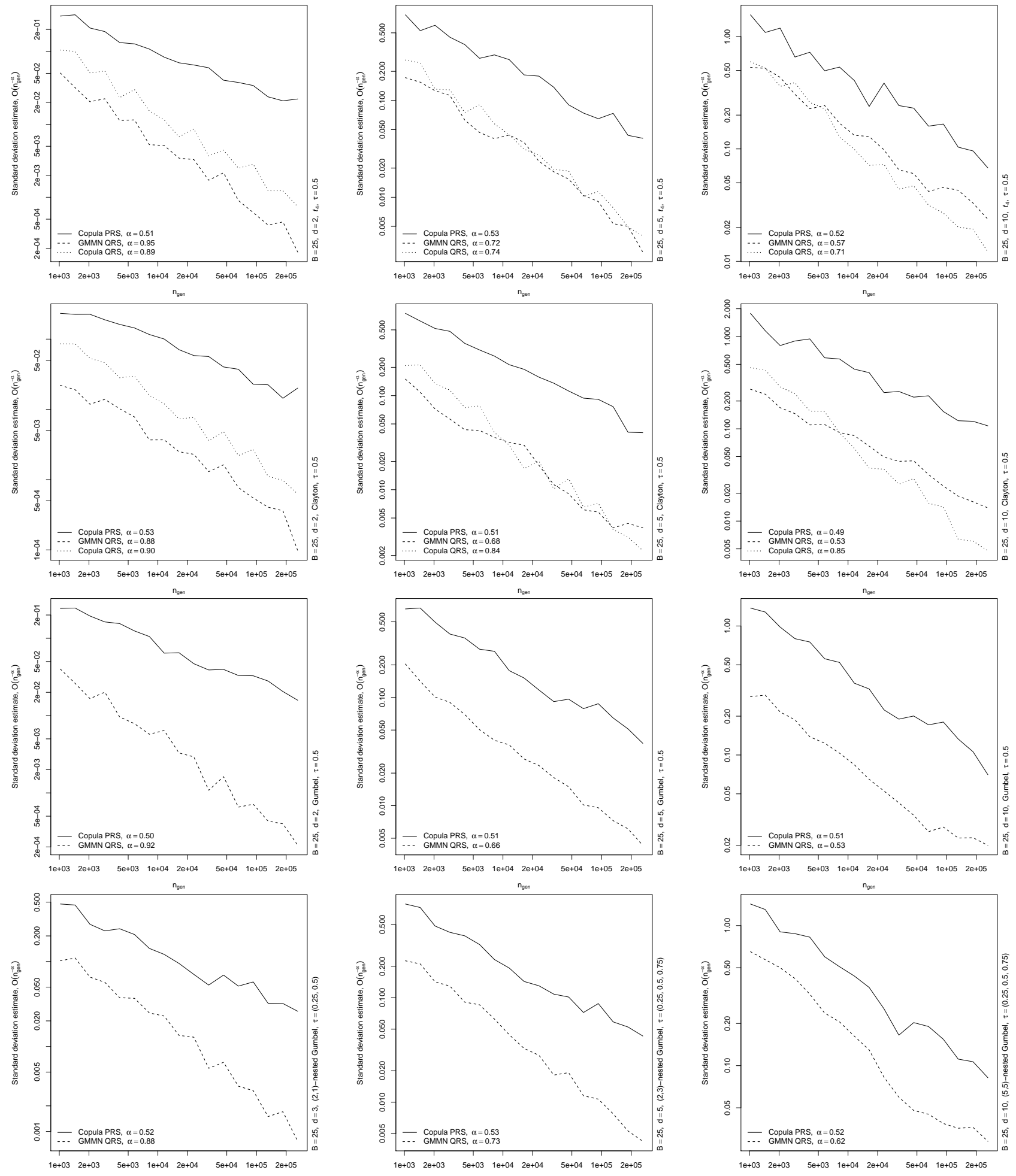

Figure 14 Standard deviation estimates based on $B=25$ replications for estimating $\mathbb{E}\left(\Psi_{2}(\boldsymbol{X})\right)$ via MC based on a pseudo-random sample (PRS), via the copula RQMC estimator (whenever available; rows 1-2 only) and via the GMMN RQMC estimator (based on digitally shifted nets). Note that in rows $1-3, d \in\{2,5,10\}$, whereas in row $4, d \in\{3,5,10\}$. 
copula. We also measured run times for $n_{\text {gen }}=10^{6}$ and $n_{\text {gen }}=10^{7}$ and they scale proportionally as one would expect.

\begin{tabular}{|c|c|c|c|c|c|c|c|c|c|}
\hline \multirow[b]{3}{*}{$d$} & \multirow[b]{3}{*}{$C$} & \multicolumn{4}{|c|}{20182.7 GHz Quad-Core Intel Core i7 } & \multicolumn{4}{|c|}{ NVIDIA Tesla P100 GPU } \\
\hline & & \multicolumn{2}{|c|}{ Copula } & \multicolumn{2}{|c|}{ GMMN } & \multicolumn{2}{|c|}{ Copula } & \multicolumn{2}{|c|}{ GMMN } \\
\hline & & PRS & QRS & PRS & QRS & PRS & QRS & PRS & QRS \\
\hline 2 & $t_{4}$ & 0.0642 & 0.4420 & 1.2960 & 1.2720 & 0.1045 & 0.8210 & 3.6140 & 3.5820 \\
\hline 2 & $\mathrm{C}$ & 0.0144 & 0.0230 & 1.3110 & 1.3140 & 0.0308 & 0.0400 & 3.6290 & 3.5820 \\
\hline 2 & $\mathrm{G}$ & 0.0348 & 374.8000 & 1.3470 & 1.3310 & 0.0669 & 687.7000 & 3.6400 & 3.5750 \\
\hline$(2,1)$ & NG & 0.0633 & NA & 1.3360 & 1.3260 & 0.1369 & NA & 3.6560 & 3.6530 \\
\hline 5 & $t_{4}$ & 0.1410 & 1.4830 & 1.4490 & 1.3830 & 0.2567 & 3.0150 & 3.7330 & 3.6960 \\
\hline 5 & $\mathrm{C}$ & 0.0425 & 0.0580 & 1.3890 & 1.5060 & 0.0936 & 0.1110 & 3.7670 & 3.6980 \\
\hline 5 & $\mathrm{G}$ & 0.0523 & 1529.5000 & 1.3930 & 1.3860 & 0.1161 & 2939.9000 & 3.7380 & 3.7010 \\
\hline$(2,3)$ & $\mathrm{NG}$ & 0.0989 & NA & 1.4020 & 1.4070 & 0.2167 & NA & 3.9450 & 3.7210 \\
\hline 10 & $t_{4}$ & 0.2766 & 3.6080 & 1.5870 & 1.6720 & 0.4917 & 6.5290 & 3.9530 & 4.0990 \\
\hline 10 & $\mathrm{C}$ & 0.0734 & 0.1190 & 1.6430 & 1.6630 & 0.1806 & 0.2320 & 3.9680 & 4.1910 \\
\hline 10 & $\mathrm{G}$ & 0.0807 & 3579.6000 & 1.5500 & 1.5290 & 0.1984 & 7119.6000 & 4.0060 & 3.9370 \\
\hline$(5,5)$ & NG & 0.1324 & NA & 1.5470 & 1.5280 & 0.3087 & NA & 3.9740 & 3.9530 \\
\hline
\end{tabular}

Table 2 Elapsed times in seconds for generating samples of size $n_{\text {gen }}=10^{5}$.

We see from Table 2 that quasi-random sampling from specific copulas is available and can be fast, e.g., for $t_{4}$ and Clayton copulas. However, we already see that quasi-random sampling gets more time-consuming for larger $d$. For other copulas, such as Gumbel copulas, it can be much more time consuming. Furthermore, as seen from the nested case and as is currently the case for most copula models, a quasi-random sampling procedure is not even available. By contrast, on the same machine, GMMNs show very close run times, are barely affected by the dimension and are not affected by the type of dependence model. For $d=10$, the GMMN quasi-random sampling procedure even outperforms the $t_{4}$ quasi-random sampling procedure for which the conditional copulas are analytically available; for $d=5$ the two procedures perform on par, depending on the machine used.

This highlights the universality of using neural networks for dependence modeling purposes. As an example, say a risk management application such as estimating expected shortfall with variance reduction is based on a $t_{4}$ copula and a regulator requires us to change the model to a Gumbel copula for stress testing purposes. Suddenly run time increases substantially. Also, if the regulator decides to incorporate hierarchies (as was more easily done for the $t_{4}$ model due to its correlation matrix) by utilizing a nested Gumbel copula, then there is suddenly no quasi-random sampling procedure known anymore. It is one of the biggest drawbacks of parametric copula models in applications that the level of difficulty of carrying out important statistical tasks such as sampling, fitting and goodness-of-fit can largely depend on the class of copulas used. These problems are eliminated with neural networks as dependence models.

\section{B.2 Fitting and training times for the real-data applications}

We now briefly present the run times for fitting the parametric copula models and training the GMMNs used in Section 5. Recall that we considered three dimensions $d \in\{3,5,10\}$ and that fitting, respectively training, was only required once for each dimension, independently of the number of applications 
considered.

Table 3 contains the elapsed times in seconds. Recall from Section 5 that GMMNs provided the best fit, followed by the unstructured $t$ copula. The latter is in general a popular parametric copula model in practice; see Fischer et al. (2009). Comparing the last two columns of Table 3 , we see that fitting the $t$ copula is comparably fast for $d=3$, however, already for $d=5$, run time for training a GMMN is on par. For $d=10$, training a GMMN is significantly faster.

\begin{tabular}{cccccrrr}
\hline$d$ & Gumbel & Clayton & Normal (ex) & Normal (un) & $t(\mathrm{ex})$ & $t(\mathrm{un})$ & GMMN \\
\hline 3 & 1.078 & 0.437 & 0.388 & 1.000 & 3.315 & 9.064 & 43.336 \\
5 & 1.291 & 0.455 & 0.435 & 6.071 & 5.932 & 41.131 & 41.235 \\
10 & 1.344 & 0.531 & 0.981 & 82.982 & 11.966 & 783.406 & 55.555 \\
\hline
\end{tabular}

Table 3 Elapsed times in seconds for fitting the respective parametric copula model and training the GMMN on one NVIDIA Tesla P100 GPU for the applications presented in Section 5.

\section{B.3 TensorFlow vs $\mathbf{R}$}

Finally, let us stress again what we initially said, namely, that run time depends on many factors. In particular, one typically relies on TensorFlow for the feed-forward step of input through the GMMN, which creates overhead especially for smaller data size $n$. In the demo GMMN_QMC_timings, we also provide a pure $\mathrm{R}$ implementation for this step for GMMNs considered in this work.

For each of $d \in\{2,5,10\}$, we randomly initialize $B=10$ GMMNs as in Algorithm 2.1 and average the elapsed times of their feed-forward steps when passing through data of size $n$ (chosen equidistant in log-scale from 10 to $10^{6}$ ) from the input distribution, once with TensorFlow, and once with our own $\mathrm{R}$ implementation. We then divide the averaged run times of the $\mathrm{R}$ implementation by the ones of the TensorFlow implementation. Whenever the ratio is smaller (larger) than one, the R implementation is faster (slower) than the TensorFlow implementation. We ran this experiment once locally on the 2018 2.7 GHz Quad-Core Intel Core i7 processor and once on the NVIDIA Tesla P100 GPU. The results are depicted on the left and on the right plot in Figure 15. respectively. Depending on the machine used, the $\mathrm{R}$ implementation can be significantly faster, especially for small $n$.

\section{References}

Arjovsky, Martin, Chintala, Soumith, and Bottou, Léon (2017), Wasserstein generative adversarial networks, International Conference on Machine Learning, 214-223.

Arora, R., Basu, A., Mianjy, P., and Mukherjee, A. (2016), Understanding deep neural networks with rectified linear units, https://arxiv.org/abs/1611.01491 (11/01/2018).

Arora, Sanjeev, Risteski, Andrej, and Zhang, Yi (2018), Do GANs learn the distribution? Some Theory and Empirics, International Conference on Learning Representations, ICLR.

Cambou, Mathieu, Hofert, Marius, and Lemieux, Christiane (2017), Quasi-random numbers for copula models, Statistics and Computing, 27(5), 1307-1329.

Constantine, G. and Savits, T. (1996), A multivariate Faa di Bruno formula with applications, Transactions of the American Mathematical Society, 348(2), 503-520.

Cranley, R. and Patterson, T. N. L. (1976), Randomization of Number Theoretic Methods for Multiple Integration, SIAM Journal on Numerical Analysis, 13(6), 904-914. 


\section{References}
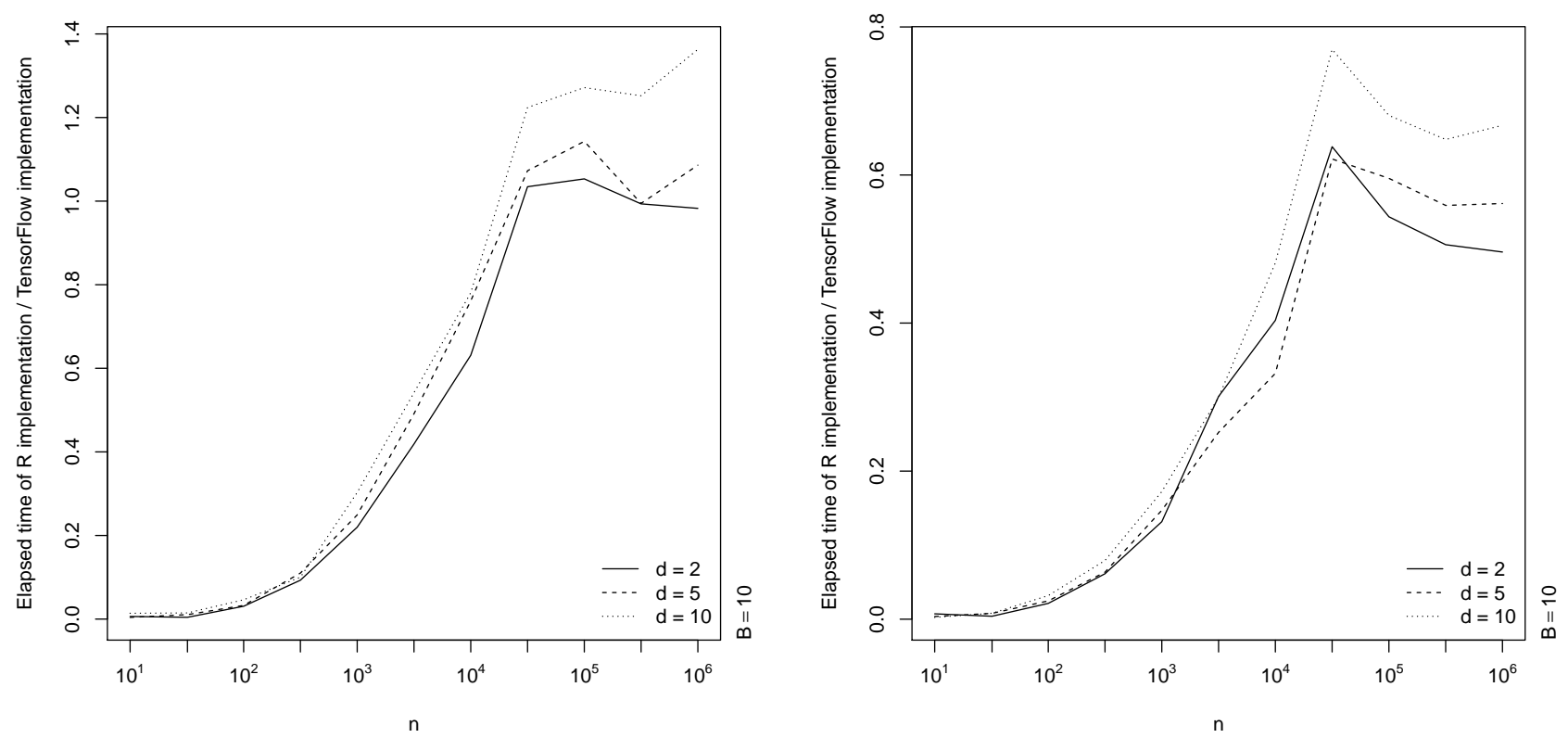

Figure 15 Ratio of averaged elapsed times of an $\mathrm{R}$ implementation over the TensorFlow implementation when evaluating randomly initialized GMMNs, once run on a $20182.7 \mathrm{GHz}$ Quad-Core Intel Core i7 processor (left) and once on an NVIDIA Tesla P100 GPU (right).

Cybenko, George (1989), Approximation by superpositions of a sigmoidal function, Mathematics of Control, Signals and Systems, 2(4), 303-314.

Dziugaite, Gintare Karolina, Roy, Daniel M, and Ghahramani, Zoubin (2015), Training generative neural networks via maximum mean discrepancy optimization, Proceedings of the Thirty-First Conference on Uncertainty in Artificial Intelligence, AUAI Press, 258-267, http://www.auai.org/ uai2015/proceedings/papers/230.pdf (08/24/2019).

E. Hlawka (1961), Über die Diskrepanz mehrdimensionaler Folgen mod 1, Mathematische Zeitschrift, $77,273-284$.

Embrechts, P., Lindskog, F., and McNeil, A. J. (2003), Modelling Dependence with Copulas and Applications to Risk Management, Handbook of Heavy Tailed Distributions in Finance, ed. by S. Rachev, Elsevier, 329-384.

Faure, H. and Lemieux, C. (2009), Generalized Halton sequence in 2008: A comparative study, ACM Transactions on Modeling and Computer Simulation, 19, Article 15.

Fischer, M., Köck, C., Schlüter, S., and Weigert, F. (2009), An empirical analysis of multivariate copula models, Quantitative Finance, 9(7), 839-854.

Genest, C., Ghoudi, K., and Rivest, L.-P. (1995), A semiparametric estimation procedure of dependence parameters in multivariate families of distributions, Biometrika, 82(3), 543-552.

Genest, Christian, Rémillard, Bruno, and Beaudoin, David (2009), Goodness-of-fit tests for copulas: A review and a power study, Insurance: Mathematics and economics, 44(2), 199-213.

Glorot, Xavier and Bengio, Yoshua (2010), Understanding the difficulty of training deep feedforward neural networks, Proceedings of the Thirteenth International Conference on Artificial Intelligence and Statistics, 249-256.

Goodfellow, Ian, Bengio, Yoshua, and Courville, Aaron (2016), Deep learning, vol. 1, MIT press Cambridge. 
Gretton, A., Borgwardt, K., Rasch, M. J., Schölkopf, B., and Smola, A. J. (2007), A kernel method for the two-sample-problem, Advances in Neural Information Processing Systems, 513-520.

Gretton, A., Borgwardt, K., Rasch, M. J., Schölkopf, B., and Smola, A. J. (2012), A kernel two-sample test, Journal of Machine Learning Research, 13(Mar), 723-773.

Hlawka, E. and Mück, R. (1972), Über eine Transformation von gleichverteilten Folgen II, Computing, $9(2), 127-138$.

Hofert, M. (2010), Sampling Nested Archimedean Copulas with Applications to CDO Pricing, PhD thesis, Südwestdeutscher Verlag für Hochschulschriften AG \& Co. KG, ISBN 978-3-8381-1656-3.

Hofert, M., Kojadinovic, I., Mächler, M., and Yan, J. (2018), Elements of Copula Modeling with R, Springer Use R! Series, ISBN 978-3-319-89635-9, doi : 10.1007/978-3-319-89635-9, http: //www . springer.com/de/book/9783319896342 (03/15/2018).

Hofert, M. and Oldford, R. W. (2018), Visualizing Dependence in High-dimensional Data: An Application to S\&P 500 Constituent Data, Econometrics and Statistics, 8, 161-183, doi:10.1016/j ecosta.2017.03.007.

Hofert, Marius (2012), A stochastic representation and sampling algorithm for nested Archimedean copulas, Journal of Statistical Computation and Simulation, 82(9), 1239-1255, doi:10.1080/ 00949655.2011 .574632 .

Hornik, Kurt (1991), Approximation capabilities of multilayer feedforward networks, Neural Networks, $4(2), 251-257$.

Joe, Harry (2014), Dependence modeling with copulas, Chapman and Hall/CRC.

Jondeau, E. and Rockinger, M. (2006), The copula-GARCH model of conditional dependencies: An international stock market application, Journal of International Money and Finance, 25, 827-853.

Kingma, Diederik P and Ba, Jimmy (2014), Adam: A method for stochastic optimization, https: //arxiv.org/abs/1412.6980 (11/01/2018).

Klambauer, Günter, Unterthiner, Thomas, Mayr, Andreas, and Hochreiter, Sepp (2017), Self-normalizing neural networks, Advances in Neural Information Processing Systems, 971-980.

L'Ecuyer, Pierre (2016), Randomized quasi-Monte Carlo: An introduction for practitioners, International Conference on Monte Carlo and Quasi-Monte Carlo Methods in Scientific Computing, Springer, 29-52.

Lemieux, Christiane (2009), Monte Carlo and Quasi-Monte Carlo Sampling, Springer.

Li, Y., Swersky, K., and Zemel, R. (2015), Generative moment matching networks, International Conference on Machine Learning, 1718-1727.

McNeil, A. J. (2008), Sampling nested Archimedean copulas, Journal of Statistical Computation and Simulation, 78(6), 567-581.

McNeil, A. J., Frey, R., and Embrechts, P. (2015), Quantitative Risk Management: Concepts, Techniques, Tools, 2nd ed., Princeton University Press.

Montufar, Guido F, Pascanu, Razvan, Cho, Kyunghyun, and Bengio, Yoshua (2014), On the number of linear regions of deep neural networks, Advances in Neural Information Processing Systems, 2924-2932.

Nelsen, R. B. (2006), An Introduction to Copulas, Springer-Verlag.

Niederreiter, Harald (1992), Random number generation and quasi-Monte Carlo methods, vol. 63, SIAM.

Nielsen, M. A. (2015), Neural Networks and Deep Learning, http://neuralnetworksanddeeplearning. com, Determination Press, (09/18/2018).

Owen, Art B (1995), Randomly permuted (t, m, s)-nets and (t, s)-sequences, Monte Carlo and Quasi-Monte Carlo Methods in Scientific Computing, Springer, 299-317. 
Owen, Art B (1997), Monte Carlo variance of scrambled net quadrature, SIAM Journal on Numerical Analysis, 34(5), 1884-1910.

Owen, Art B (2003), Variance and discrepancy with alternative scramblings, ACM Transactions of Modeling and Computer Simulation, 13(4).

Owen, Art B (2008), Local antithetic sampling with scrambled nets, The Annals of Statistics, 36(5), 2319-2343.

Pascanu, Razvan, Mikolov, Tomas, and Bengio, Yoshua (2013), On the difficulty of training recurrent neural networks, International Conference on Machine Learning, 1310-1318.

Patton, A. J. (2006), Modelling Asymmetric Exchange Rate Dependence, International Economic Review, 47(2), 527-556, http://public.econ.duke.edu/ ap172/Patton_IER_2006.pdf.

Rémillard, Bruno and Scaillet, Olivier (2009), Testing for equality between two copulas, Journal of Multivariate Analysis, 100(3), 377-386.

Rosenblatt, Murray (1952), Remarks on a multivariate transformation, The Annals of Mathematical Statistics, 23(3), 470-472.

Sobol', Il'ya Meerovich (1967), On the distribution of points in a cube and the approximate evaluation of integrals, Zhurnal Vychislitel'noi Matematiki i Matematicheskoi Fiziki, 7(4), 784-802.

Tolstikhin, Ilya O, Gelly, Sylvain, Bousquet, Olivier, Simon-Gabriel, Carl-Johann, and Schölkopf, Bernhard (2017), Adagan: Boosting generative models, Advances in Neural Information Processing Systems, 5424-5433. 